\title{
tRNAs as regulators of biological processes
}

\author{
Medha Raina ${ }^{1,2}$ and Michael lbba ${ }^{1,2}$ * \\ 1 Department of Microbiology, The Ohio State Biochemistry Program, The Ohio State University, Columbus, OH, USA \\ ${ }^{2}$ Center for RNA Biology, The Ohio State University, Columbus, OH, USA
}

\section{Edited by:}

Akio Kanai, Keio University, Japan

Reviewed by:

Tohru Yoshihisa, University of Hyogo, Japan

Dieter Soll, Yale University, USA

\section{*Correspondence:}

Michael Ibba, Department of Microbiology, The Ohio State

Biochemistry Program, The Ohio State University, 484 West 12th Avenue,

Columbus, OH 43210-1292, USA

e-mail: ibba.1@osu.edu

\begin{abstract}
Transfer RNAs (tRNA) are best known for their role as adaptors during translation of the genetic code. Beyond their canonical role during protein biosynthesis, tRNAs also perform additional functions in both prokaryotes and eukaryotes for example in regulating gene expression. Aminoacylated tRNAs have also been implicated as substrates for nonribosomal peptide bond formation, post-translational protein labeling, modification of phospholipids in the cell membrane, and antibiotic biosyntheses. Most recently tRNA fragments, or tRFs, have also been recognized to play regulatory roles. Here, we examine in more detail some of the new functions emerging for tRNA in a variety of cellular processes outside of protein synthesis.
\end{abstract}

Keywords: amino acid, protein synthesis, regulation, transfer RNA, translation

\section{INTRODUCTION}

tRNAs are important players in the protein synthesis pathway, linking the genetic code with the amino acid sequence of proteins. tRNAs are composed of 73-90 nucleotides and have a characteristic cloverleaf secondary structure made up of the D-loop, T loop, variable loop, and the anticodon loop. The tRNA further folds into an L-shaped tertiary structure through coaxial stacking of the T and $\mathrm{D}$ loops. To function as a substrate in protein synthesis, tRNA is charged with an amino acid by its cognate aminoacyl-tRNA synthetase. The aminoacyl-tRNA (aa-tRNA) thus formed serves as a substrate and participates in the chemistry of peptide bond formation in the process of protein synthesis. Beside this well-known canonical role during protein biosynthesis, tRNAs have been shown to perform additional functions such as acting as signaling molecules in the regulation of numerous metabolic and cellular processes in both prokaryotes and eukaryotes. Aminoacylated tRNAs have also been implicated as substrates for non ribosomal peptide bond formation in the case of cell wall formation, protein labeling for degradation, modification of phospholipids in the cell membrane, and antibiotic biosynthesis. Due to their universally conserved L-shaped three-dimensional conformation, which is stabilized by extensive secondary and tertiary structural contacts and modifications, tRNA molecules are among the most stable RNAs in a cell and are considerably more robust than mRNAs (Gebetsberger and Polacek, 2013). For a long time, tRNA fragments were considered as non-functional degradation intermediates, but have now been recognized to be major RNA species in human cells for which regulatory roles are beginning to be discovered. It was also recently shown that tRNAs can act as an effective scavenger of cytochrome $c$, consistent with a role in regulating apoptosis. With new functions still emerging for tRNA, in this review we examine some of the many "non-protein synthesis" roles of tRNA in the cell.

\section{ROLES OF tRNA IN GENE EXPRESSION}

While aminoacyl-tRNAs have been implicated in many roles outside translation, several important functions of tRNA have been found not to require the aminoacyl form (aa-tRNA). Uncharged tRNAs have been shown to regulate global gene expression in response to changes in amino acid pools in the cell. Bacteria have adopted various strategies to adapt to external stresses, of which the most-studied global regulatory system is the stringent response. Stringent response is mediated through the production of the alarmone $5^{\prime}$-diphosphate $3^{\prime}$-diphosphate guanosine (ppGpp) and $5^{\prime}$-triphosphate $3^{\prime}$-diphosphate guanosine (pppGpp) which were first discovered by Cashel and Gallant (1969) in Escherichia coli as a response to amino acid starvation. E. coli uses two pathways for the synthesis of ppGpp dependent on RelA and SpoT. RelA is a ribosome-associated (p)ppGpp synthase which senses the presence of uncharged tRNAs that accumulate at the ribosome A site as a result of amino acid limitation. The presence of the uncharged tRNA acts as an effector molecule, stalling protein synthesis and activating RelA which then synthesizes pppGpp and ppGpp by phosphorylation of GTP or GDP using ATP as the phosphate donor (Haseltine and Block, 1973; Sy and Lipmann, 1973). ppGpp was recently shown to bind at an interface of $\omega$ and $\beta^{\prime}$ subunits of RNA polymerase, thereby acting as an allosteric effector to inhibit global gene transcription, while stimulating the expression of only a few genes related to the synthesis of amino acids (Ross et al., 2013). rRNA and tRNA synthesis are primarily inhibited, resulting in the global downregulation of bacterial metabolism. SpoT is a bifunctional (p)ppGpp synthase and hydrolase, which presumably regulates the (p)ppGpp level in response to nutrient deficiency. The mechanism by which SpoT senses starvation and synthesizes ppGpp is unclear (Magnusson et al., 2005). Many other bacterial species including Bacillus subtilis contain only one RelA-SpoT homolog, designated as Rel, which possesses both (p)ppGpp synthase and hydrolase activities. RelA-SpoT homologs have also been detected in plants (Givens et al., 2004). Two Bacillus subtilis genes, yjbM and $y w a C$, were found to encode a novel (p)ppGpp synthase that corresponds to the synthase domain of RelA-SpoT family members while having a different mode of action (Nanamiya et al., 2008). 
Another mechanism by which bacteria regulate gene expression using uncharged tRNA as the effector molecule has been demonstrated in B. subtilis and other Gram-positive bacteria. In these organisms, the expression of aminoacyl-tRNA synthetase genes and genes involved in amino acid biosynthesis and uptake is regulated by the T box control system (reviewed in Green et al., 2010). Regulation by the $\mathrm{T}$ box mechanism most commonly occurs at the level of transcription attenuation (Henkin and Yanofsky, 2002). The $5^{\prime}$ untranslated regions of regulated genes contain a 200$300 \mathrm{nt}$ conserved sequence and structural element (a G + C-rich helix followed by a run of $U$ residues) that serves as an intrinsic transcriptional terminator and can also participate in formation of an alternate, less stable antiterminator structure. During amino acid starvation, binding of a specific uncharged tRNA stabilizes the antiterminator and in doing so prevents formation of the terminator helix. The $\mathrm{T}$ box binds specific uncharged tRNA at two conserved sites: the anticodon of the tRNA interacts with the codon sequence of the specifier loop (SL) in the $5^{\prime}$-UTR, while the $3^{\prime}$ acceptor end interacts with the UGGN sequence found in the antiterminator bulge, thus stabilizing the structure of the antiterminator and preventing the formation of the competing terminator. RNA polymerase then continues past the terminator region and transcribes the full-length mRNA. The $\mathrm{N}$ residue in the antiterminator bulge varies with the corresponding position of the tRNA. Both charged and uncharged tRNAs can interact with specifier sequence in the $5^{\prime}$-UTR; however the presence of the amino acid at the $3^{\prime}$ end of a charged tRNA prevents the interaction of its $3^{\prime}$ end with the antiterminator bulge region; and allows formation of the terminator hairpin that results in premature termination of transcription (Grundy et al., 2005). Recently a unique mechanism of tRNA-dependent regulation at the transcriptional level was discovered. Saad etal. (2013) found a two-codon Tbox riboswitch binding two tRNAs in Clostridium acetobutylicum. This T-box regulates the operon for the essential tRNA-dependent transamidation pathway and harbors an SL with two potential overlapping codon positions for tRNA ${ }^{\text {Asn }}$ and tRNA ${ }^{\text {Glu }}$. Both tRNAs can efficiently bind the SL in vitro and in vivo. This feature allows the riboswitch to sense two tRNAs and balance the biosynthesis of two amino acids (Saad et al., 2013). Regulation at the level of translation initiation has also been demonstrated for $\mathrm{T}$ box riboswitches in certain bacteria (Fuchs et al., 2006). Translationally regulated leader RNAs include an RNA element with the ability to sequester the Shine-Dalgarno (SD) sequence by pairing with a complementary anti-SD (ASD) sequence. Binding of uncharged tRNA stabilizes a structure analogous to the antiterminator that includes the ASD sequence, and formation of this alternate structure releases the SD sequence for binding of the $30 \mathrm{~S}$ ribosomal subunit, thereby enabling translation of mRNA coding for proteins involved in amino acid biosynthesis (Green et al., 2010).

Uncharged tRNAs also function as regulators in eukaryotes. In amino-acid-starved yeast and mammalian cells, uncharged tRNA activates a protein kinase named Gcn2p whose regulatory sequences include the amino terminal region, a pseudo kinase domain, protein kinase region, histidyl-tRNA synthetase (HisRS)related region and the c-terminal dimerization and ribosome binding sequences. The tRNA has been shown to bind to the
HisRS like regulatory domain, thereby activating Gcn2p which in turn phosphorylates eIF2, a protein involved in binding GTP and Met-tRNA ${ }_{i}{ }^{\text {Met }}$ and forming the ternary complex required for translation initiation (Wek et al., 1995). The activated Gcn2p phosphorylates the $\alpha$ subunit of eIF 2 at serine 51 , lowering its activity and thereby reduces global protein synthesis. Gcn2p was shown to bind several types of uncharged tRNA with similar affinities but showed a reduced affinity for the charged form of a tRNA, implying that Gcn 2 can discriminate between charged and uncharged forms of tRNA. (Dong et al., 2000). It was recently proposed that in the inactive form of Gcn 2 present in non-starvation conditions, association with the substrate eIF2 is prevented by binding of the HisRS-like domain and $\mathrm{C}$-term to the PK domain of Gcn 2 thereby sequestering the substrate binding cleft. However, under starvation conditions, uncharged tRNA binds to Gcn2, at both the HisRS and $\mathrm{C}$-term domains thereby producing conformational changes which open up the substrate binding cleft in the PK domain by releasing the HisRS-like domain and the C-terminal portion of Gcn2p, from inhibitory interactions with the PK domain, which allows eIF2 binding and phosphorylation (Qiu et al., 2001).

It has been proposed that discrimination between the charged and uncharged tRNA by Gcn2p occurs via an analogous mechanism of RelA protein activation as observed in E. coli by the presence of uncharged tRNA at the decoding (A) site on translating ribosomes. The activation of Gcn $2 p$ by uncharged tRNA requires its association with the ribosome via its $\mathrm{C}$-terminal region and also, interactions between the $\mathrm{N}$ terminus of Gcn $2 \mathrm{p}$ and the Gcn1p-Gcn20p protein complex which is also associated with the ribosome. Gcnlp, has been proposed to facilitate the eviction of uncharged tRNA from the A site and its transfer from the A site to the HisRS-like domain in Gcn2p for kinase activation and the Gcn1p-Gcn20p complex has also been implicated to increase the binding of uncharged tRNA to ribosomes. The importance of the Gcn1p-Gen20p complex in Gcn2p activation was shown by the Hinnebusch group, who demonstrated that deletion of GCN1 blocks eIF2 phosphorylation by Gen2p (Marton et al., 1993). The activation of eIF2 by an uncharged tRNA at the A site of the ribosome could explain how starvation of a single amino acid can activate Gcn2p, even though it cannot discriminate between uncharged tRNA species in cells starved for only one amino acid (Marton et al., 1997; Garcia-Barrio et al., 2000; Sattlegger and Hinnebusch, 2000). In yeast, phosphorylation of eIF2, allows for selected mRNAs such as GCN4 to be translated. Elevated levels of Gcn4, which acts as a transcription factor, stimulate the expression of genes involved in amino acid biosynthesis (reviewed in Hinnebusch, 2005). In comparison to S. cerevisiae, which has a single eIF2 $\alpha$ kinase, Gcn 2 p, mammalian cells have expanded this stress response pathway to include additional eIF $2 \alpha$ kinases, which each respond to different environmental stresses. Analogous to yeast, phosphorylation of mammalian eIF2 $\alpha$ leads to a block in global translation, accompanied by induced translational expression of ATF4 and ATF5, transcription factors related to Gcn4p (Harding et al., 2000; Lu et al., 2004; Vattem and Wek, 2004; Zhou et al., 2008).

The above mechanisms demonstrate that under certain nutritional stresses, the aminoacylation levels of tRNAs change and the 


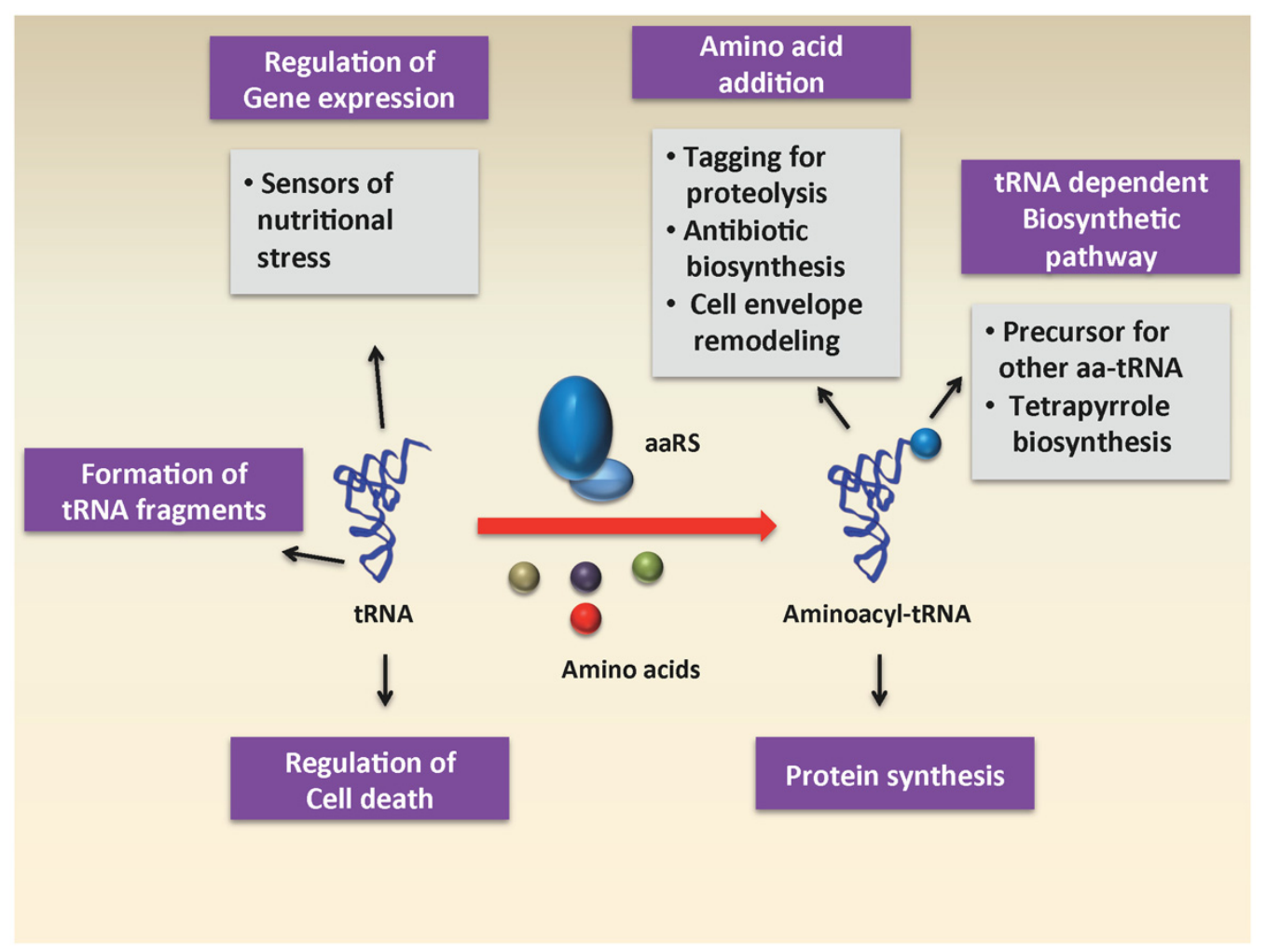

FIGURE 1 | Various roles of charged and uncharged tRNA in the cell.

accumulated uncharged tRNAs participate in numerous biological pathways that regulate global gene expression levels, helping the organism to survive under adverse conditions.

\section{AMINOACYL-tRNAs AS NON-RIBOSOMAL SUBSTRATES}

In recent years, the diverse roles of aa-tRNAs have received a great deal of attention. While much of the research has focused on the use of aa-tRNA by the ribosome for protein synthesis, a number of studies have uncovered roles for aa-tRNAs as substrates in other biochemical processes, such as cell wall formation, protein labeling for degradation, aminoacylation of phospholipids in the cell membrane, and antibiotic biosynthesis (Figure 1). In this section, we will briefly review some of these various processes that use aa-tRNAs as substrates.

\section{AMINOACYL-tRNAs IN CELL WALL BIOGENESIS Aminoacyl-tRNA-dependent building of peptidoglycan bridges}

Peptidoglycans (PG) are structural components of bacterial cell walls that can both serve as a barrier to environmental challenges and provide a scaffold for the attachment of various proteins including virulence factors (Vollmer et al., 2008). Peptidoglycan is a polymer of $\beta$ (1-4)-linked $N$-acetylglucosamine (GlcNAc) and $N$-acetylmuramic acid (MurNAc), with all lactyl groups of MurNAc substituted with stem peptides, typically comprised of alternating $\mathrm{D}$ and L-amino acids with an overall common structure of L-Ala- $\gamma$-D-Glu-X-D-Ala-D-Ala. The composition of the peptide varies among different bacteria: Gram-negative bacteria and Gram-positive bacilli have meso-diaminopimelic acid (DAP) as the third amino acid (DAP-type peptidoglycan), whereas most other Gram-positive bacteria (including Grampositive cocci) have L-lysine as the third amino acid (Royet and Dziarski, 2007). The stem peptides from adjacent strands are often crosslinked, either directly or through short peptides between the $\mathrm{X}$ position of the first pentapeptide side chain with the LAla at the fourth position of another. The amino acids required for bridge formation are typically derived from aminoacylatedtRNA donor molecules and are transferred onto the pentapeptide by tRNA-dependent aminoacyl-ligases which catalyze peptidebond formation by using aminoacyl-tRNAs and peptidoglycan precursors as donor and acceptor, respectively.

The peptidoglycan in Streptococcus pneumoniae contains a "stem peptide" composed of up to five amino acids, Ala- $\gamma$-D-GluLys-D-Ala-D-Ala, with an L-Ala-L-Ala or an L-Ser-L-Ala dipeptide branch that is attached to the third L-Lys of the pentapeptide side chain. MurM is responsible for the addition of either L-Ala or L-Ser as the first amino acid of the cross-link and then MurN invariably adds L-Ala as the second amino acid (Filipe et al., 2000). In both cases, appropriately aminoacylated-tRNA species serve as the amino acid donors for the reaction (Lloyd et al., 2008), although MurM also efficiently accepts mischarged tRNA substrates (Shepherd, 2011; Shepherd and Ibba, 2013b). In Enterococcus faecalis, BppA1 and BppA2 add L-Ala-L-Ala dipeptide to the pentapeptide chain (Bouhss et al., 2002), while FemXAB from Staphylococcus aureus sequentially adds one (FemX) or two (FemA 
and FemB) glycines (Schneider et al., 2004). Lif and Epr in Staphylococcus simulans and Staphylococcus capitis, FemX in Weissella virides and FemX and VanK in Streptomyces coelicolor all catalyze similar reactions using aa-tRNAs as substrates (reviewed in Shepherd and Ibba, 2013a). How aa-tRNAs are diverted from protein synthesis and used as substrates by these enzymes remains somewhat unclear in most instances. In S. aureus, the mechanism of escape from the protein synthesis machinery could be explained by the observation that three out of the five tRNA ${ }^{\text {Gly }}$ isoacceptors encoded in the S. aureus genome have sequence identity elements consistent with weak binding to EF-Tu (Giannouli et al., 2009). These specific tRNA sequence elements include replacement of the strong EF-Tu binding pairs G49-U65 and G51-C63 [23-25] with A49-U65 and A51-U63, respectively, in the T loop (Roy etal., 2007; Sanderson and Uhlenbeck, 2007). The three non-proteinogenic tRNA ${ }^{\text {Gly }}$ isoacceptors also show replacement of GG at positions 18 and 19 with either UU or CU. Hence the isoacceptors with weak binding to EFTu could escape protein synthesis and thus allow S. aureus to maintain an adequate supply of Gly-tRNA ${ }^{\text {Gly }}$ for two essential processes: translation and cell wall modification (Shepherd and Ibba, 2013a).

The specificity of peptidoglycan-modifying enzymes with respect to amino acid and tRNA substrates was demonstrated in the Fem X enzyme from Weissella viridescens. In W. viridescens the peptide bridge is made up of L-Ala-L-Ser or L-Ala-L-SerL-Ala. FemX initiates peptide bridge formation by transfer of the first L-Ala residue to the amino group of L-Lys found at the third position of the pentapeptide side chain. The enzymes involved in the subsequent transfer of the second position Ser and third position Ala residues have not yet been identified. FemX has a preference for L-Ala addition to UDP-MurNAc pentapeptide because it reacts much more unfavorably with both L-Ser and the acceptor arm of tRNA Gly. In vitro assays show that FemX turns over Ser-tRNA ${ }^{\text {Ser }}$ and Gly-tRNA ${ }^{\text {Gly }}$ 17- and 38fold less efficiently than Ala-tRNA ${ }^{\text {Ala }}$, respectively. In the latter case, the penultimate base pair of tRNA ${ }^{\text {Ala }}$, G2-C71, was identified as an essential identity element for FemX. This is typically replaced by C2-G71 in tRNA ${ }^{\text {Gly }}$ species (Fonvielle et al., 2009). L-Ala is preferred 110-fold over D-Ala, suggesting relatively weak specificity toward different stereoisomers. The exclusion of ser-

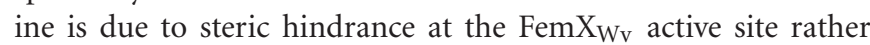
than poor recognition of the nucleotide sequence of tRNA ${ }^{\mathrm{Ser}}$. Hence, Fem enzymes discriminate non-cognate aa-tRNAs on the basis of both the aminoacyl moiety and the sequence of the tRNA.

\section{Aminoacyl-tRNA-dependent aminoacylation of membrane lipids}

Bacteria are frequently exposed to cationic antimicrobial peptides (CAMPs), for example eukaryotic host defense peptides or prokaryotic bacteriocins, whose cationic properties impart strong affinities to the negatively charged bacterial lipids phosphatidylglycerol (PG) and cardiolipin (CL). Many bacteria, among them several important human pathogens, achieve CAMP resistance using MprF proteins, a unique group of enzymes that aminoacylate anionic phospholipids with L-lysine or L-alanine, thereby introducing positive charges into the membrane surface and reducing the affinity for CAMPs (Ernst and Peschel, 2011). MprF was first identified when its inactivation rendered a $S$. aureus transposon mutant susceptible to a wide range of cationic antimicrobial peptides (CAMPs) leading to the name "multiple peptide resistance factor" (MprF; Peschel etal., 2001). MprFs can use lysyl or alanyl groups derived from aminoacyl tRNAs for modification of PG (Roy and Ibba, 2008). MprF proteins are integral membrane proteins made up of a $\mathrm{C}$ terminal, hydrophilic, cytoplasmic domain responsible for the transfer of amino acid onto PG, and an $\mathrm{N}$ terminal transmembrane hydrophobic domain that flips newly synthesized LysPG to the membrane outer leaflet (Ernst et al., 2009). MprF homologs can be found in most bacterial phylas and are abundant in firmicutes, actinobacteria, and proteobacteria with the exception of enterobacteria. Some archaea also harbor genes for MprF, probably resulting from lateral gene transfer events (Roy and Ibba, 2009). MprF homologs exhibit differential specificity for the aa-tRNA substrate they use to modify PG, resulting in a broader classification of these enzymes as aminoacylphosphatidylglycerol synthases (aaPGS; Klein etal., 2009; Dare and Ibba, 2012). For example, the MprFs in S. aureus and P. aeruginosa only synthesize Lys-PG or Ala-PG, respectively (Staubitz etal., 2004; Klein etal., 2009). In contrast, Enterococcus faecium MprF2 exhibits rather relaxed specificity for the donor substrate and produces both, Ala-PG and Lys-PG along with small amounts of Arg-PG (Roy etal., 2009). Listeria monocytogenes $\mathrm{MprF}$ is less strict in its specificity for the acceptor substrate and generates both, Lys-PG and LysCL (Thedieck etal., 2006; Dare etal., 2014). Based on the ability of MprF1 to efficiently recognize tRNA ${ }^{\mathrm{Ala}}$, $\mathrm{tRNA}^{\mathrm{Pro}}$, and a minihelix ${ }^{\text {Ala }}$ and recognition of the tRNA ${ }^{\text {Lys }}$ species from both Borrelia burgdoferi and humans, which share less than $50 \%$ sequence identity, it was proposed that the specificity of $\mathrm{MprF}$ arises from direct recognition of the aminoacyl moiety of aa-tRNA (Roy and Ibba, 2008). The mechanism utilized by MprF and other similar enzymes raises the question of how aa-tRNA donor substrates are directed into membrane lipid modification and away from protein synthesis. Determination of the $K_{\mathrm{D}}$ s of Lys-tRNA for EF-Tu and for MprF suggested that the two proteins have similar affinities for tRNA under physiological conditions (Roy and Ibba, 2008). Comparison of the sites in tRNA recognized by MprF and EF-Tu would give a better understanding of how aa-tRNAs are partitioned between translation and membrane lipid modification pathways.

\section{ROLE OF AA-tRNA IN ANTIBIOTIC BIOGENESIS}

In addition to having essential roles in protein synthesis and nonribosomal peptide bond formation, aminoacyl-tRNAs are also used in pathways where the donated amino acid moiety undergoes transformation into a significantly different compound. These pathways involve different amino acid-tRNA pairs and a variety of acceptor molecules (Banerjee et al., 2010). Examples of aatRNA-dependent addition of amino acids in antibiotic biogenesis, which have been reviewed in detail previously, include valanimycin, pacidamycin, and cyclodipeptide synthesis (Shepherd and Ibba, 2013a). 
Valanimycin is a potent antitumor and antibacterial azoxy compound first isolated from Streptomyces viridifaciens by Yamato et al. (1986). A gene cluster has been identified that contains 14 genes involved in the biosynthesis of valanimycin (Garg et al., 2002). The functions of the products of eight of these genes have now been established. Valanimycin is derived from L-Val and L-Ser via an isobutylhydroxylamine intermediate. VlmD, VlmH, and VlmR catalyze the conversion of valine into isobutylhydroxylamine, while VlmL catalyzes the formation of L-seryl-tRNA from L-serine. VlmA, which is a homolog of the housekeeping SerRS, catalyzes the transfer of L-serine from L-seryl-tRNA to isobutylhydroxylamine, to produce $O$-(L-seryl)-isobutylhydroxylamine, while VlmJ and VlmK catalyze the phosphorylation and subsequent dehydration of the biosynthetic intermediate valanimycin hydrate to form valanimycin (Garg and Parry, 2010). The mechanism by which Ser-tRNA ${ }^{\text {Ser }}$ is directed away from translation into the valanimycin pathway, and the identity elements of tRNA $^{\text {Ser }}$ that help in recognition by VlmA and VlmL, are still unknown.

Other examples of antibiotics derived from aa-tRNAs are the cyclodipeptides (CDP), a large group of secondary metabolites with a notable range of clinical activities (Rodriguez and Carrasco, 1992; Prasad, 1995; Magyar et al., 1996; Waring and Beaver, 1996; Kanoh etal., 1999; Strom etal., 2002; Cain etal., 2003; Kanzaki et al., 2004; Jia et al., 2005; Kohn and Widger, 2005; Musetti etal., 2007; Minelli etal., 2012). It was originally proposed that formation of the CDPs was catalyzed by nonribosomal peptide synthetases, which do not use aa-tRNAs as substrates. However, subsequent characterization of synthesis of the CDP albonoursin in Streptomyces noursei identified the tRNA-dependent CDP synthase AlbC (Lautru et al., 2002). AlbC synthesizes the albonoursin precursor cyclo (L-Phe-L-Leu) from aminoacylated tRNAs in an ATP-independent reaction (Lautru etal., 2002; Gondry et al., 2009). CDP synthase products identified to date include cyclo(L-Leu-L-Leu) (cLL), cyclo(L-Phe-LLeu) (cFL), cyclo(L-Tyr-L-Tyr) (cYY), and cyclo(L-Trp-L-Xaa) (cWX), all of which are intermediates in antibiotic synthesis (Belin etal., 2012). CDP synthases use their two aa-tRNA substrates in a sequential ping-pong mechanism, with a similar first catalytic step: the binding of the first aa-tRNA and subsequent transfer of its aminoacyl moiety to the conserved serine residue of the enzyme pocket (e.g., Ser37 in the AlbC enzyme; Sauguet etal., 2011). The mechanism of addition of the second amino acid remains unclear, as do the specificity determinants for CDP synthases. Recently, similarities between the predicted secondary structure for $\mathrm{PaCB}$, a protein involved in the biosynthesis of the antibiotic pacidamycin, and structures of two Fem transferases led to the characterization of PacB as an alanyl-tRNA-dependent transferase (Zhang et al., 2011). Pacidamycins are a family of uridyl tetra/pentapeptide antibiotics produced by Streptomyces coeruleorubidus with antipseudomonal activities through inhibition of the translocase MraY during bacterial cell wall assembly. Analogous to the activity of CDP synthases, PacB hijacks aa-tRNAs and transfers L-Ala from aminoacyl-tRNA donors to the $\mathrm{N}$ terminal $\mathrm{m}-\mathrm{Tyr}_{2}$ residue of the growing PacH-anchored antibiotic scaffold (Zhang et al., 2010).

\section{tRNA-DEPENDENT ADDITION OF AMINO ACIDS TO THE AMINO-TERMINUS OF PROTEINS}

Protein degradation plays an important role in maintaining cellular physiology and in regulation of various cellular processes such as cell growth, differentiation and apoptosis by removing damaged polypeptides and regulatory proteins in a timely manner. As compared to cellular compartments like lysosomes and vacuoles where proteases are involved in non-specific degradation of proteins, protein degradation in the cytosol of prokaryotes and eukaryotes is often strictly targeted to protect cellular proteins from unwanted degradation. One means to achieve specificity involves the aa-tRNA transferases, which recognize a secondary destabilizing residue (pro- $\mathrm{N}$ degrons) at the $\mathrm{N}$-terminus of a target peptide and utilize an aminoacyl-tRNA to transfer a primary destabilizing amino acid ( $\mathrm{N}$-degron) to the $\mathrm{N}$-terminal residue, making the protein a target for the cellular degradation machinery (N-recognins; Mogk et al., 2007). This specificity in protein degradation was discovered by Bachmair et al. (1986) when they found that different genetic constructs of $\beta$-galactosidase proteins from E. coli exhibited very different half-lives when produced in Saccharomyces cerevisiae, ranging from more than $20 \mathrm{~h}$ to less than 3 min, depending on the identity of their N-terminal amino acid [the $\mathrm{N}$-end rule (Bachmair et al., 1986)]. The $\mathrm{N}$-end rule relates the identity of the $\mathrm{N}$-terminal residue of a protein to its in vivo half-life (Mogk et al., 2007) and has been shown to function in bacteria (Tobias et al., 1991), fungi (Bachmair et al., 1986), plants (Potuschak et al., 1998) and mammals (Gonda et al., 1989). In eukaryotes an $\mathrm{N}$-terminal Arg residue is the preferred $\mathrm{N}$-degron and acts as a target for ubiquitin conjugation and subsequent degradation by the eukaryotic proteasome (Tasaki et al., 2012). The degron is generated by the ATE1 gene product arginyl $(R)$-transferase, which transfers Arg from Arg-tRNA to the Nterminal $\alpha$-amino group of oxidized cysteine, Asp, or Glu which constitute secondary destabilizing residues (Pro-N-degrons; Rai and Kashina, 2005; Graciet etal., 2006). In prokaryotes, Leu and Phe act as the primary destabilizing $\mathrm{N}$-terminal residues $(\mathrm{N}$ degrons) and can be generated by two classes of aa-transferases, leucyl/phenylalanyl(L/F)-transferase encoded by the Aat gene and leucyl- transferase encoded by Bpt. The L/F- transferase attaches a primary destabilizing residue of either Leu or Phe to the secondary destabilizing residues Lys, Met, and Arg (Shrader et al., 1993), whereas Bpt-encoded L-transferase attaches Leu to the secondary destabilizing residues Asp and Glu (Graciet et al., 2006). The Leu/Phe N-degron acts as a target for ClpS, which transfers the protein to ClpAP for subsequent degradation (Mogk et al., 2007). The question that next arises is how the aa-tRNA transferases achieve specificity in binding aa-tRNAs? The crystal structure of leucyl/phenylalanyl-tRNA-protein transferase and its complex with an aminoacyl-tRNA analog solved by Suto et al. (2006) revealed that the side chain of Leu or Phe is accommodated in a highly hydrophobic pocket, with a shape and size suitable for hydrophobic amino-acid residues lacking a branched $\beta$-carbon, such as leucine and phenylalanine. The adenosine group of the $3^{\prime}$ end of tRNA is recognized largely through $\pi-\pi$ stacking with conserved Trp residues. However, L/F transferases achieve specificity for aa-tRNAs through specific interaction with the aminoacyl moiety and not the tRNA, and only the presentation of the specific 
aminoacyl moiety by a single-stranded RNA region is required for recognition (Abramochkin and Shrader, 1996). The activity of $\mathrm{L} / \mathrm{F}$-transferases is reduced in the presence of an excess of EF-Tu, suggesting that L/F-transferase and EF-Tu compete for binding to aa-tRNA.

\section{tRNA-DERIVED FRAGMENTS}

Small non-coding RNA (sncRNA) molecules are major contributors to regulatory networks that control gene expression, and significant attention has been directed toward their identification and studying their biological functions. sncRNA was first discovered in 1993 in Caenorhabditis elegans, and since then a large number of sncRNAs have been identified. sncRNAs are 16-35 nucleotides (nts) long and are classified into different groups such as microRNA (miRNA), small-interfering RNA (siRNA), piwiinteracting RNA, and small nucleolar RNA (snoRNA). Among them, miRNA and siRNA are the most extensively studied, and both suppress gene expression by binding to target mRNAs. The recent development of high-throughput sequencing technology has improved the identification of other types of small, RNAs-like, tRNA-derived RNA fragments (tRFs) which have been identified by several research groups (Lee et al., 2009). There is increasing evidence that these are not by-products from random degradation, but rather functional molecules that can regulate translation and gene expression. The production of tRNA fragments and their emerging roles in the cell are discussed below (Figure 2).

\section{PRODUCTION OF tRNA FRAGMENTS tRNA halves}

tRNA halves are composed of 30-35 nucleotides derived from either the $5^{\prime}$ or $3^{\prime}$ part of full-length, mature tRNA. These tRNA halves are produced by cleavage in the anticodon loop under nutritional, biological, physicochemical, or oxidative stress (Thompson et al., 2008; Dhahbi et al., 2013; Nowacka et al., 2013). In mammalian cells, tRNA halves are generated during stress conditions by the action of the nuclease angiogenin, a member of the RNase A family (Fu et al., 2009) whereas in yeast Rnylp, a member of the RNase T2 is responsible for tRNA half production. Apart from their roles as nucleases, both angiogenin and Rnyl act as sensors of cellular damage and can promote cell death and inhibit tumor formation (Thompson and Parker, 2009; Gebetsberger and Polacek, 2013). Under normal conditions, yeast Rny1 is usually localized in the vacuole (Thompson and Parker, 2009), while angiogenin is secreted to the plasma, sequestered in the nucleolus

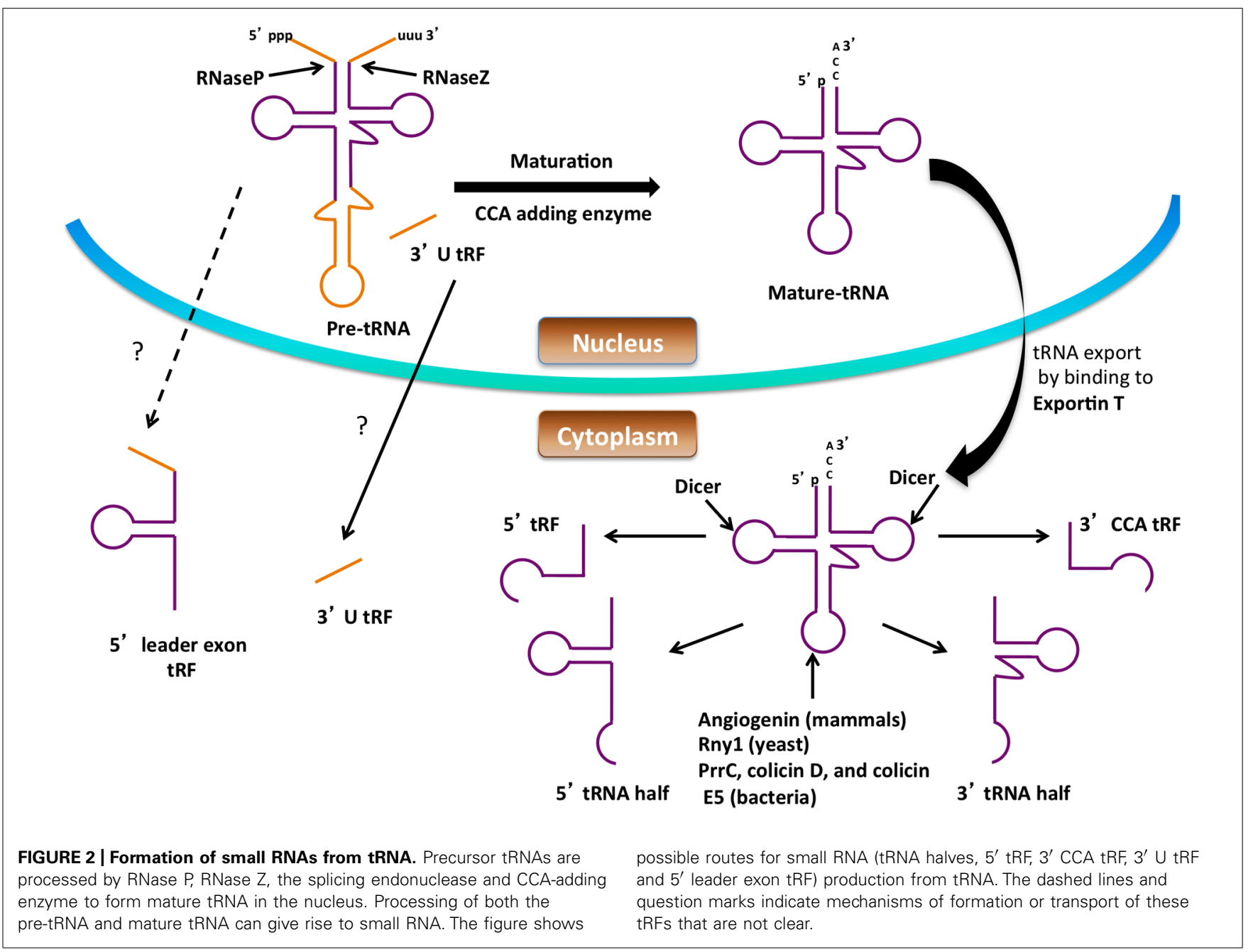


or bound to its inhibitor RNH1 (Yamasaki et al., 2009; Saikia et al., 2012), and released into the cytoplasm under certain stress conditions. tRNA halves have also been identified in bacteria, archaea, and plants. In bacteria, tRNA anticodon nucleases such as PrrC, colicin D, and colicin E5 have been shown to cleave specific subsets of tRNAs (reviewed in Kaufmann, 2000; Masaki and Ogawa, 2002).

\section{tRNA-derived fragments}

tRNA-derived fragments (tRFs) are shorter than tRNA halves ranging between 13 and $20 \mathrm{nt}$ in size. They have been identified in all domains of life. There are four types of tRFs known and they are classified based on the part of the mature tRNA or pre-tRNA from which they are derived. tRFs were classified as $5^{\prime}$ tRFs, $3^{\prime}$ CCA tRFs, $3^{\prime} \mathrm{U}$ tRFs, or $5^{\prime}$ leader-exon tRFs. $5^{\prime}$ tRFs are derived from the $5^{\prime}$ end of the tRNA generated at any point of tRNA processing, provided the $5^{\prime}$ leader sequence is removed by RNaseP, and are formed by a cleavage in the D loop. In the case of $5^{\prime}$ tRFs their biogenesis is carried out by Dicer in mammalian cells (Cole et al., 2009). However, it is known that the Dicer-independent generation of $5^{\prime}$ tRFs takes place in Schizosaccharomyces pombe due to the differences in length of the $5^{\prime}$ tRFs generated in these two organisms (19 nt long in mammals and $23 \mathrm{nt}$ long in yeast), suggesting that a protein other than Dicer is responsible for their production in yeast (Buhler et al., 2008). 3' CCA tRFs are produced from the $3^{\prime}$ ends of mature tRNA by cleavage at the T loop and carry the trinucleotide CCA at the acceptor stem. Dicer has been implicated in the generation of the $3^{\prime}$ end fragment (Maute et al., 2013), although angiogenin and other RNase A members have also been proposed to function in Dicer-independent processing (Li et al., 2012; Gebetsberger and Polacek, 2013). 3’ U tRFs are cleaved from the $3^{\prime}$ end of tRNA precursors by RNase $\mathrm{Z}$, and their biogenesis is normally Dicer independent. They commonly start directly after the $3^{\prime}$ end of mature tRNAs and end in a stretch of $\mathrm{U}$ residues produced by RNA polymerase III run-off (Lee et al., 2009; Haussecker et al., 2010). One 3' U tRF is produced in an RNaseZ-independent manner by the action of Dicer on the predicted bulged hairpin structure of the pre-tRNA (Babiarz et al., 2008). The mechanism of formation of $5^{\prime}$ leader-exon tRFs is not known; however they have been identified in CLP1 mutant cells possibly arising due to aberrant splicing. CLP1 is an RNA kinase and is a component of the mRNA $3^{\prime}$ end cleavage and polyadenylation machinery in mammals (Hanada et al., 2013).

While it was previously thought that production of tRNA halves and tRFs were solely mechanisms to remove damaged tRNAs, increasing evidence suggests their formation to be regulated. Angiogenin and Rnyl involved in the production of tRNA halves are usually sequestered in compartments before they are released in the cytoplasm where they cleave tRNAs (Spriggs et al., 2010). However, the regulation of their release from these cellular compartments is not known. Also a number of tRNAs [including tRNA $^{\text {Asp(GTC) }}$, tRNA ${ }^{\mathrm{Val}(\mathrm{AAC})}$ and tRNA ${ }^{\mathrm{Gly}(\mathrm{GCC})}$ ] can be methylated by Dnmt2, which has been shown to protect these tRNAs from cleavage during stress (Schaefer et al., 2010). This specificity in cleavage of tRNAs might be responsible for the different types of tRFs observed under various conditions.

\section{FUNCTIONS OF TRFS}

Are tRFs merely the products of tRNA degradation or do they have bona fide biological functions? If so, how diverse are these functions given the various forms of tRFs identified? Several lines of evidence point toward regulated production, suggesting that they may be functional RNA species. First, the abundance of different types of tRF does not correlate with the number of parent tRNA gene copies (Kawaji et al., 2008; Cole et al., 2009; Hsieh et al., 2009; Sobala and Hutvagner, 2011) with the exception of those found in Tetrahymena (Couvillion et al., 2010). Second, the fragments of tRNA formed are produced by cleavage at specific points in the tRNA. Third, whilst tRFs corresponding to the $5^{\prime}$ and $3^{\prime}$ ends of tRNA have been reported, those corresponding to the middle (incorporating the anticodon loop) have not. Although, the exact roles of tRNA halves and tRFs are yet to be elucidated, accumulating evidence suggests that tRNA-derived small RNAs participate in two main types of biological processes as discussed in more detail below.

\section{Translation regulation of gene expression under stress conditions}

tRNAs are indispensible components of the translational machinery, hence tRNA cleavage under stress conditions can affect protein synthesis. However, the mode of translational regulation by tRNA cleavage is not simple. It has been shown previously that during stress conditions, formation of tRNA cleavage products does not change the pool of full-length tRNA significantly, rather these fragments represent only a small portion of the tRNA pool (Saikia et al., 2012). Ivanov et al. (2011) showed a more intricate role for tRNA halves in translational control. They observed that tRNA halves formed by angiogenin during stress were able to inhibit protein synthesis and trigger the phospho-eIF2 $\alpha$-independent assembly of stress granules (SGs). These granules are mainly composed of stalled pre-initiation complexes, suggesting that the translation initiation machinery can be targeted by 5 ' tRNA halves. They demonstrated that selected tRNA halves inhibit protein synthesis by displacing eIF4G/eIF4A from capped and uncapped mRNA and eIF4E/G/A (eIF4F) from the $\mathrm{m}^{7} \mathrm{G}$ cap. Using pull down of $5^{\prime}$-tiRNA ${ }^{\mathrm{Ala}}$ - protein complexes the authors implicated YB-1, a translational repressor known to displace eIF4G from RNA and eIF4E/G/A from the $\mathrm{m}^{7} \mathrm{G}$ cap (Evdokimova et al., 2001; Nekrasov etal., 2003). Analysis of the $5^{\prime}$ tRNA halves in complex with YB-1 revealed that a terminal oligo-G motif containing four to five consecutive guanosines present in certain $5^{\prime}$ tRNA halves (Ala/Cys) was absolutely required for translational repression of a reporter mRNA, suggesting the inhibition is caused by specific tRNA and is not a consequence of global upregulation of tRFs (Ivanov et al., 2011). This result came as a surprise as regulation of translation during stress is carried out via phosphorylation of eIF2 (See Roles of tRNA in Gene Expression), which induces translational repression facilitated by active sequestration of untranslated mRNAs into SGs (Holcik and Sonenberg, 2005).

In addition to tRNA halves, tRFs have also been implicated in regulation of translation. In the archaeon Haloferax volcanii a 26 nt-long $5^{\prime}$ tRF originating from tRNA ${ }^{\mathrm{Val}}$ in a stress-dependent manner was shown to directly bind to the small ribosomal subunit and inhibit translation by interfering with peptidyl transferase 
activity (Gebetsberger et al., 2012). A similar mechanism of translation inhibition by a $5^{\prime} \mathrm{tRF}$ was recently observed in human cells (Sobala and Hutvagner, 2013). A 26 nt 5' tRF derived from tRNA $^{\mathrm{Val}}$ was able to inhibit translation by affecting peptide bond formation. An interesting observation from this study was that the tRFs required a conserved "GG" dinucleotide for their activity in inhibiting translation. A similar motif dependence is observed as discussed above in translation inhibition by a $5^{\prime}$ tRNA half. $5^{\prime}$ tRNA halves containing the $5^{\prime}$ tRF sequence were shown to require a run of at least four guanosine residues at the $5^{\prime}$ end of the molecule, which is present only in $\mathrm{tRNA}^{\mathrm{Ala}}$ and $\mathrm{tRNA}{ }^{\mathrm{Cys}}$, as compared to $5^{\prime}$ tRFs that require only two guanosine residues at the $3^{\prime}$ end of the molecule, residues conserved between tRNAs. Mutating the di-guanosine motif required by $5^{\prime} \mathrm{tRF}$ in the $5^{\prime}$ tRNA half did not affect its inhibitory activity, and the precise mechanism of translation inhibition by these tRFs warrants further investigation (Sobala and Hutvagner, 2013).

\section{tRNA-derived fragments as regulators of gene silencing}

One of the first studies showing the involvement of tRNA-derived fragments in gene regulation and silencing was carried out by Yeung et al. who addressed the role of small RNAs in human immunodeficiency virus (HIV) infected cells. A highly abundant, 18 nt-long, tRF originating from the $3^{\prime}$ end of human cytoplasmic tRNA ${ }^{\text {Lys } 3}$ was shown to target the the HIV-1 primer-binding site (PBS) similarly to siRNAs that target complementary RNA (Yeung et al., 2009). tRNA ${ }^{\text {lys }}$ is used by viral reverse transcriptases as primer for the initiation of reverse transcription and DNA synthesis (Marquet et al., 1995). The $3^{\prime}$ tRF was shown to be associated with Dicer and AGO2, and to cause RNA cleavage of the complementary PBS sequence thereby showing the role of a tRF in viral gene silencing. Other tRFs like $3^{\prime} \mathrm{CCA}$, $5^{\prime}$ and $5^{\prime} \mathrm{U}$ tRF have also been shown to be associated with agronautes and hence have a potential to function as an siRNA or miRNA. Haussecker etal. (2010) investigated the ability of $3^{\prime} \mathrm{CCA}$ and $3^{\prime} \mathrm{U}$ tRFs to associate with Argonaute proteins and cause silencing of a reporter luciferase transgene. They found that both types of $3^{\prime}$ tRF associated with Argonaute proteins, but often more effectively with the non-silencing Ago3 and Ago4 than Ago1 or Ago2. They observed that $3^{\prime}$ CCA tRFs had a moderate effect on reporter transgene silencing, but $3^{\prime} \mathrm{U}$ tRFs did not. However, upon co-transfection of a small RNA complementary to the $3^{\prime} \mathrm{U}$ tRF, the tRF preferentially associated with Ago 2 and caused $80 \%$ silencing of the reporter transgene. This correlated with redirection of the reconstituted fully duplexed double-stranded RNA into Ago 2, whereas Ago 3 and 4 were skewed toward less structured small RNAs, particularly singlestranded RNAs. This is in stark contrast with results normally obtained in the miRNA field where sequences complementary to miRNAs relieve repression, a phenomenon known as senseinduced transgene silencing (SITS). Modulation of tRF levels had minor effects on the abundance of microRNAs, but more pronounced changes in the silencing activities of both microRNAs and siRNAs. This study provides compelling evidence that tRFs play a role in the global control of small RNA silencing through associating with different Argonaute proteins (Haussecker et al., 2010).
A tRF that functions as an miRNA was recently described, a $22 \mathrm{nt} 3^{\prime}$ tRF generated in a Dicer-dependent manner from tRNA Gly in mature B cells and associated with Argonaute proteins (Maute et al., 2013). The $3^{\prime}$ tRF was shown to inhibit RPA1, an essential gene involved in DNA repair by possibly binding to the $3^{\prime}$ UTR region. Expression of this tRF was downregulated in a lymphoma cell line indicating that loss of $3^{\prime} \mathrm{tRF}$ expression might help the cancer cells to tolerate the accumulation of mutations and genomic aberrations during tumor progression.

\section{Other biological functions of tRFs}

Apart from the two known biological functions of tRFs in regulation, other potential biological functions are beginning to be identified. Recently a study by Ruggero et al. (2014) showed their role in viral infectivity. Large scale sequencing of small RNA libraries was used to identify small non-coding RNAs expressed in normal $\mathrm{CD}^{+}{ }^{+} \mathrm{T}$ cells compared to cells transformed with human T-cell leukemia virus type 1 (HTLV-1), the causative agent of adult T-cell leukemia/lymphoma (ATLL). Among the miRNAs and tRFs expressed, one of the most abundant tRFs found was derived from the $3^{\prime}$ end of tRNA ${ }^{\text {Pro }}$, and exhibited perfect sequence complementarity to the primer binding site of HTLV-1. In vitro reverse transcriptase assays verified that this tRF was capable of priming HTLV-1 reverse transcriptase thereby suggesting an important role in viral infection. One possible role suggested for the tRF fragment is to support the initiation of reverse transcription, but not progressivity, with failure to proceed to the strand transfer step (Ruggero et al., 2014). Further studies are now needed to compare the abilities of the tRF and of full-length $\mathrm{tRNA}^{\text {Pro }}$ to prime and support strand transfer. Variation of tRNA halves accumulation was also shown in the parasites Toxoplasma gondii, the agent of toxoplasmosis, and the rodent malaria parasite Plasmodium berghei. These organisms exhibited increased tRNA accumulation upon egress from host cells and in response to stage differentiation, amino acid starvation, and heat-shock. It was observed that avirulent isolates of $T$. gondii and attenuated $P$. berghei parasites displayed higher rates of tRNA cleavage compared to virulent strains. Also tRNA half production was significantly higher in the metabolically quiescent bradyzoite and sporozoite stages of $T$. gondii, compared to the fast-growing tachyzoite indicating a relationship between half-tRNA production and growth rate in this important group of organisms (Galizi et al., 2013). A role for tRF halves in Respiratory Syncytial Virus (RSV) infectivity was recently shown by Wang et al. who observed an induction of tRNA cleavage upon RSV infection with a specific subset of tRNAs being cleaved. The $31 \mathrm{nt} 5^{\prime} \mathrm{tRF}(\mathrm{Glu})$ formed exhibited trans-silencing capability against target genes; however the mechanism of gene silencing was found to be different than the gene-silencing mechanism of miRNA/siRNA, previously also shown for other tRFs. Interestingly the tRF was also shown to promote RSV replication (Wang et al., 2013)

tRNA fragments have also been implicated in progressive motor neuron loss. Hanada et al. recently demonstrated that tRNA fragments generated in CLP1 mutant cells sensitize cells to oxidative stress-induced activation of the p53 tumor suppressor pathway and in turn lead to progressive loss of spinal motor neurons leading to muscle denervation and paralysis thereby providing a possible 
link between tRNA cleavage and p53 dependent cell death. However, the exact mechanism by which these tRNA fragments affect the p53 pathway needs to be determined (Hanada et al., 2013).

\section{REGULATION OF CELL DEATH BY tRNA}

Apoptosis is a cellular process by which damaged, harmful, and unwanted cells are eliminated. Apoptotic regulation is critical to cell homeostasis, immunity, multi-cellular development, and protection against infections and diseases like cancer (Thompson, 1995). Apoptotic cells have been shown to undergo various morphological and biochemical changes caused by a group of cysteine-dependent aspartate specific proteases, or caspases. In healthy cells, caspases are inactive, however during apoptosis caspases are activated and signal the onset of apoptosis via cleavage of various intracellular proteins including apoptotic proteins, cellular structural and survival proteins, transcriptional factors, signaling molecules, and proteins involved in DNA and RNA metabolism (Li and Yuan, 2008; Hou and Yang, 2013). Cleavage of these intracellular proteins ultimately leads to phagocytic recognition and engulfment of the dying cell. While many factors have been discovered that regulate the apoptotic pathway, in this section the recently discovered role of tRNA as a regulator of cell death is discussed (Figure 3).

\section{CASPASE ACTIVATION BY EXTRINSIC AND INTRINSIC PATHWAYS}

Apoptosis can be triggered via two major routes: an extrinsic, or extracellularly activated pathway and/or an intrinsic, or mitochondrial-mediated pathway. Both pathways activate caspases, a class of endoproteases that hydrolyze peptide bonds (Thornberry and Lazebnik, 1998). Although there are various types of caspases, those involved in apoptosis can be classified into two groups, the initiator (or apical) caspases and the effector (or executioner) caspases. Initiator caspases (e.g., Caspases-8 and 9) are capable of autocatalytic activation, whereas effector caspases (e.g., Caspases-3, 6 and 7) are activated by initiator caspase cleavage (Chang and Yang, 2000; Riedl and Shi, 2004). The extrinsic pathway begins outside the cell through activation of a group of pro-apoptotic cell surface receptors, such as Fas/CD95 and tumor necrosis factor receptor. Upon binding to their cognate ligand, these receptors recruit an adaptor protein Fas-associated death domain (FADD) that binds and dimerizes the initiator procaspase- 8 , to form an oligomeric death-inducing signaling complex (DISC), in which procaspase8 becomes activated through an autoproteolytic cleavage event. The active caspase- 8 then cleaves and activates the effector caspases 3 and 7 (Ashkenazi and Dixit, 1998; Krammer et al., 2007; Hou and Yang, 2013). The intrinsic pathway causes mitochondrial outer membrane permeabilization (MOMP), which leads to release of cytochrome $c$, a mitochondrial protein which transfers electrons from complex III to complex IV in the electron transport chain (Wang, 2001). The discovery of the role of cytochrome $c$ in apoptosis by Liu etal. (1996) came as a surprise due to its essential role in the survival of the cell. In the cytosol, cytochrome $c$ interacts with the apoptotic protease activating factor-1 (APAF-1) to form the apoptosome complex (Zou et al., 1997). The complex recruits procaspase-9, which converts to active caspase- 9 by autocatalysis. Active caspase9 activates effector caspases like caspase-7 and caspase- 3 and causes apoptosis (Figure 3). Apoptosis is regulated by several pro-apoptotic proteins (Bax, Bak, and Bid), anti-apoptotic proteins (Bcl-2, Bcl- $\mathrm{X}_{\mathrm{L}}$, and $\left.\mathrm{Mcl}-1\right)$ and a range of cellular factors (HSP90, HSP70 and HSP27; Sreedhar and Csermely, 2004; Gorla and Sepuri, 2014) that is now known to include tRNA.

\section{INTERACTION BETWEEN tRNA AND CYTOCHROME c: POTENTIAL ROLE IN REGULATING APOPTOSIS}

To answer the long-standing conundrum of why $1 \mathrm{mM}$ dATP is required to induce caspase- 9 activation in cell lysates, when the intracellular concentration of dATP is only $10 \mu \mathrm{M}$, Mei et al. investigated the role of RNA, which is essentially a polymer of nucleoside monophosphates, in cytochrome $c$-mediated caspase activation. They observed that treatment of mammalian S100 extracts with RNase strongly increased cytochrome $c$-induced caspase- 9 activation, while the addition of RNA to the extracts impaired caspase- 9 activation. These results implicated an inhibitory role of RNA in the activation of caspase-9. Systematic evaluation of the steps leading to caspase-9 activation identified cytochrome $c$ as the target of the RNA inhibitor. Analysis of cytochrome $c$-associated species revealed that tRNA binds specifically to cytochrome $c$. Microinjection of tRNA into living cells inhibited the ability of cytochrome $c$ to induce apoptosis, while degradation of tRNA by an RNase that preferentially degrades tRNA, onconase, enhanced apoptosis via the intrinsic pathway. Taken together, these findings showed that tRNA binds to cytochrome $c$ and inhibits formation of the apoptosome (Mei et al., 2010). This suggested a direct role for tRNA in regulating apoptosis and revealed an intimate connection between translation and cell death. This finding also raised an interesting question as to how the interaction between tRNA and cytochrome $c$ modulates apoptosis. This question was addressed recently by Gorla et al. who proposed that tRNA interacts with the heme moiety of cytochrome $c$ and thereby protects the positively charged surface of cytochrome $c$ from being exposed to the APAF-1 complex. This model was further confirmed by the observation that cytochrome $c$ lost its ability to interact with tRNA after treatment with oxidizing agents or cysteine modifying agents. In such a state, hemin is unable to bind to tRNA and the exposed positively charged residues of cytochrome $c$ then bind to APAF-1 (Gorla and Sepuri, 2014). Hence tRNA can regulate apoptosis by binding to cytochrome $c$. Further investigation of the nucleotide residues of tRNA involved in these interactions is required to answer questions about how tRNA binding to cytochrome $c$ is regulated in the cell, whether specific tRNA isoacceptors are involved, and if this interaction is non-specific. Increased expression of tRNA has been detected in a wide variety of transformed cells (Marshall and White, 2008), such as ovarian and cervical cancer (Winter et al., 2000; Daly et al., 2005), carcinomas, and multiple myeloma cell lines (Zhou et al., 2009). Expression levels of tRNA molecules in breast cancer cells were 10-fold higher as compared to in normal cells and overexpression of $\mathrm{tRNA}_{\mathrm{i}}{ }^{\mathrm{Met}}$ induces proliferation and immortalization of fibroblasts and also significantly alters the global 


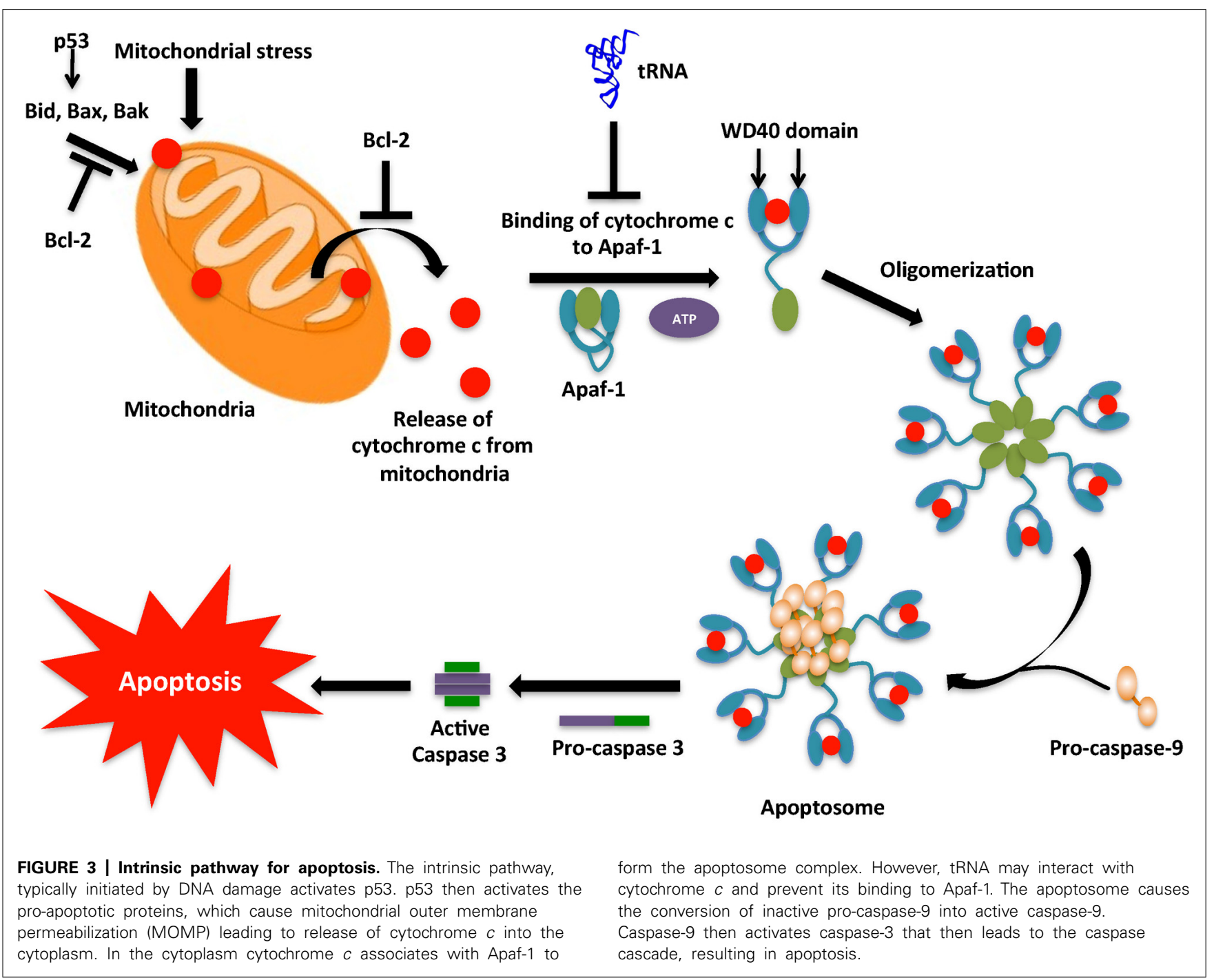

tRNA expression profile (Pavon-Eternod et al., 2013). It was also observed that certain individual tRNAs were overexpressed

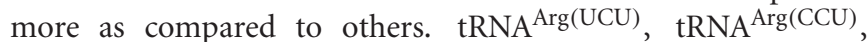
tRNA $^{\text {Thr(CGU) }}$ tRNA $^{\text {Ser(CGA) }}$, and tRNA ${ }^{\text {Tyr(GTA) }}$ were among the most over-expressed tRNAs, while tRNA ${ }^{\text {His(GTG) }}$ tRNA $^{\text {Phe(GAA), }}$ and tRNA $^{\text {Met(CAT) }}$ were the least over-expressed tRNAs (Pavon-Eternod etal., 2009) indicating overexpression is not random and may be related to regulation of cytochrome $c$. Identification of the tRNA sites involved in binding to cytochrome $c$ might help elucidate the connection between tRNA overexpression and cancer. tRNA cleavage has also been suggested as a mode of regulation of this interaction (Hou and Yang, 2013).

\section{CONCLUSION}

While aa-tRNAs have been implicated in variety of roles in biosynthetic pathways, much less is known about the various functions of uncharged tRNAs in cells apart from their role in acting as sensors for cellular stress like nutritional deprivation. The recent discovery of the role of tRNA in regulating apoptosis has opened a whole new field which requires investigation into tRNA-protein interactions and has created a link between regulation of cell death and cellular metabolism. With the advent of high throughput sequencing techniques, studying the whole transcriptome of various organisms has become feasible. These techniques refuted the age-old assumption that rRNA, mRNA and tRNA constitute the main RNA species in the cell. It is now clear that almost all of the DNA in the cell is transcribed; however, only a small portion of these transcripts are translated into proteins or used as substrates for biological processes. The emergence of these sequencing techniques has resulted in discoveries of novel ncRNAs, and several studies have highlighted their role as important regulators of gene expression. Among the ncRNAs discovered, a number of cleavage products of tRNAs formed in response to stress have been also been discovered. These cleavage products were initially thought to be a result of random degradation; however, a number of studies have revealed their production to be a result of specific cleavage, and possibly regulated. Although a number of cleavage products have been observed, all the possible mechanisms of their production are not fully understood. 
Also in the case of $3^{\prime} U$ tRF and $5^{\prime}$ leader exon tRF that are most likely produced inside the nucleus, their mechanism of export from the nucleus is not understood. Also regulation of tRNA fragment production, i.e., its initiation, efficiency, and termination are not fully understood. A recent study by Hanada et al. (2013) demonstrated that tRNA fragments sensitize cells to oxidative stress-induced activation of the p53 tumor suppressor pathway. This suggests that tRNA cleavage activates apoptosis via activation of p53 and hence protects against cancer, while full-length tRNA binds cytochrome $c$ and prevents apoptosis thereby aiding cancer development. This hypothesis is strengthened by the overexpression of tRNAs observed in cancer cell lines. Further investigation into the link between tRNA cleavage and p53 activation is required to help understand how tRNAs help regulate the progression of cancer.

tRNA is post-transcriptionally modified at various nucleotides. While their role in tRNA structure stability and translation is well studied, these modifications might aid in the regulation of tRNA fragmentation. Further studies are needed to answer why some tRNAs are cleaved and others not - for example could modifications make certain positions in tRNA more sensitive to RNases or could they be responsible for blocking RNases? Also, modifications might also help regulate tRNA binding to cytochrome $c$ during apoptosis. Regulation of this interaction and its role in metabolism and tumorigenesis will help our understanding of regulation of death in both normal and cancer cells. Cells have various mechanisms to sense the absence of a modification and remove non-functional tRNAs (Phizicky and Alfonzo, 2010). Variations in the modification status of tRNAs during stress have been implicated directly in decoding (Dedon and Begley, 2014), and such effects may be accentuated by indirect effects on the generation of regulatory tRFs. Clearly, much still remains to be discovered about the various regulatory roles of both charged and uncharged tRNA.

\section{ACKNOWLEDGMENTS}

Work in the authors' laboratory on this topic was supported by grants MCB 1052344 from the National Science Foundation and GM65183 from the National Institutes of Health.

\section{REFERENCES}

Abramochkin, G., and Shrader, T. E. (1996). Aminoacyl-tRNA recognition by the leucyl/phenylalanyl-tRNA-protein transferase. J. Biol. Chem. 271, 22901-22907. doi: 10.1074/jbc.271.37.22901

Ashkenazi, A., and Dixit, V. M. (1998). Death receptors: signaling and modulation. Science 281, 1305-1308. doi: 10.1126/science.281.5381.1305

Babiarz, J. E., Ruby, J. G., Wang, Y., Bartel, D. P., and Blelloch, R. (2008). Mouse ES cells express endogenous shRNAs, siRNAs, and other microprocessorindependent, Dicer-dependent small RNAs. Genes Dev. 22, 2773-2785. doi: 10.1101/gad.1705308

Bachmair, A., Finley, D., and Varshavsky, A. (1986). In vivo half-life of a protein is a function of its amino-terminal residue. Science 234, 179-186. doi: 10.1126/science.3018930

Banerjee, R., Chen, S., Dare, K., Gilreath, M., Praetorius-Ibba, M., Raina, M., et al. (2010). tRNAs: cellular barcodes for amino acids. FEBS Lett. 584, 387-395. doi: 10.1016/j.febslet.2009.11.013

Belin, P., Moutiez, M., Lautru, S., Seguin, J., Pernodet, J. L., and Gondry, M. (2012). The nonribosomal synthesis of diketopiperazines in tRNAdependent cyclodipeptide synthase pathways. Nat. Prod. Rep. 29, 961-979. doi: $10.1039 / \mathrm{c} 2 \mathrm{np} 20010 \mathrm{~d}$
Bouhss, A., Josseaume, N., Severin, A., Tabei, K., Hugonnet, J. E., Shlaes, D., et al. (2002). Synthesis of the L-alanyl-L-alanine cross-bridge of Enterococcus faecalis peptidoglycan. J. Biol. Chem. 277, 45935-45941. doi: 10.1074/jbc.M207449200

Buhler, M., Spies, N., Bartel, D. P., and Moazed, D. (2008). TRAMP-mediated RNA surveillance prevents spurious entry of RNAs into the Schizosaccharomyces pombe siRNA pathway. Nat. Struct. Mol. Biol. 15, 1015-1023. doi: 10.1038/nsmb.1481

Cain, C. C., Lee, D., Waldo, R. H. III, Henry, A. T., Casida, E. J. Jr., Wani, M. C., et al. (2003). Synergistic antimicrobial activity of metabolites produced by a nonobligate bacterial predator. Antimicrob. Agents Chemother. 47, 2113-2117. doi: 10.1128/AAC.47.7.2113-2117.2003

Cashel, M., and Gallant, J. (1969). Two compounds implicated in the function of the RC gene of Escherichia coli. Nature 221, 838-841. doi: 10.1038/221838a0

Chang, H. Y., and Yang, X. (2000). Proteases for cell suicide: functions and regulation of caspases. Microbiol. Mol. Biol. Rev. 64, 821-846. doi: 10.1128/MMBR.64.4.821846.2000

Cole, C., Sobala, A., Lu, C., Thatcher, S. R., Bowman, A., Brown, J. W., et al. (2009). Filtering of deep sequencing data reveals the existence of abundant Dicer-dependent small RNAs derived from tRNAs. RNA 15, 2147-2160. doi: 10.1261/rna.1738409

Couvillion, M. T., Sachidanandam, R., and Collins, K. (2010). A growth-essential Tetrahymena Piwi protein carries tRNA fragment cargo. Genes Dev. 24, 27422747. doi: 10.1101/gad.1996210

Daly, N. L., Arvanitis, D. A., Fairley, J. A., Gomez-Roman, N., Morton, J. P., Graham, S. V., et al. (2005). Deregulation of RNA polymerase III transcription in cervical epithelium in response to high-risk human papillomavirus. Oncogene 24, 880888. doi: 10.1038/sj.onc. 1208031

Dare, K., and Ibba, M. (2012). Roles of tRNA in cell wall biosynthesis. Wiley Interdiscip. Rev. RNA 3, 247-264. doi: 10.1002/wrna.1108

Dare, K., Shepherd, J., Roy, H., Seveau, S., and Ibba, M. (2014). LysPGS formation in Listeria monocytogenes has broad roles in maintaining membrane integrity beyond antimicrobial peptide resistance. Virulence 5, 534-546. doi: 10.4161/viru. 28359

Dedon, P. C., and Begley, T. J. (2014). A system of RNA modifications and biased codon use controls cellular stress response at the level of translation. Chem. Res. Toxicol. 27, 330-337. doi: 10.1021/tx400438d

Dhahbi, J. M., Spindler, S. R., Atamna, H., Yamakawa, A., Boffelli, D., Mote, P., et al. (2013). 5' tRNA halves are present as abundant complexes in serum, concentrated in blood cells, and modulated by aging and calorie restriction. BMC Genomics 14, 298. doi: 10.1186/1471-2164-14-298

Dong, J., Qiu, H., Garcia-Barrio, M., Anderson, J., and Hinnebusch, A. G. (2000). Uncharged tRNA activates GCN2 by displacing the protein kinase moiety from a bipartite tRNA-binding domain. Mol. Cell 6, 269-279. doi: 10.1016/S10972765(00)00028-9

Ernst, C. M., and Peschel, A. (2011). Broad-spectrum antimicrobial peptide resistance by MprF-mediated aminoacylation and flipping of phospholipids. Mol. Microbiol. 80, 290-299. doi: 10.1111/j.1365-2958.2011. 07576.x

Ernst, C. M., Staubitz, P., Mishra, N. N., Yang, S. J., Hornig, G., Kalbacher, H., et al. (2009). The bacterial defensin resistance protein MprF consists of separable domains for lipid lysinylation and antimicrobial peptide repulsion. PLoS Pathog. 5:e1000660. doi: 10.1371/journal.ppat.10006

Evdokimova, V., Ruzanov, P., Imataka, H., Raught, B., Svitkin, Y., Ovchinnikov, L. P., etal. (2001). The major mRNA-associated protein YB-1 is a potent $5^{\prime}$ cap-dependent mRNA stabilizer. EMBO J. 20, 5491-5502. doi: 10.1093/emboj/20.19.5491

Filipe, S. R., Pinho, M. G., and Tomasz, A. (2000). Characterization of the murMN operon involved in the synthesis of branched peptidoglycan peptides in Streptococcus pneumoniae. J. Biol. Chem. 275, 27768-27774. doi: 10.1074/jbc.M004675200

Fonvielle, M., Chemama, M., Villet, R., Lecerf, M., Bouhss, A., Valéry, J. M., et al. (2009). Aminoacyl-tRNA recognition by the FemXWv transferase for bacterial cell wall synthesis. Nucleic Acids Res. 37, 1589-1601. doi: 10.1093/nar/gkn1039

Fu, H., Feng, J., Liu, Q., Sun, F., Tie, Y., Zhu, J., et al. (2009). Stress induces tRNA cleavage by angiogenin in mammalian cells. FEBS Lett. 583, 437-442. doi: 10.1016/j.febslet.2008.12.043

Fuchs, R. T., Grundy, F. J., and Henkin, T. M. (2006). The S(MK) box is a new SAM-binding RNA for translational regulation of SAM synthetase. Nat. Struct. Mol. Biol. 13, 226-233. doi: 10.1038/nsmb1059 
Galizi, R., Spano, F., Giubilei, M. A., Capuccini, B., Magini, A., Urbanelli, L., et al. (2013). Evidence of tRNA cleavage in apicomplexan parasites: half-tRNAs as new potential regulatory molecules of Toxoplasma gondii and Plasmodium berghei. Mol. Biochem. Parasitol. 188, 99-108. doi: 10.1016/j.molbiopara.2013.03.003

Garcia-Barrio, M., Dong, J., Ufano, S., and Hinnebusch, A. G. (2000). Association of GCN1-GCN20 regulatory complex with the N-terminus of eIF2alpha kinase GCN2 is required for GCN2 activation. EMBO J. 19, 1887-1899. doi: 10.1093/emboj/19.8.1887

Garg, R. P., Ma, Y., Hoyt, J. C., and Parry, R. J. (2002). Molecular characterization and analysis of the biosynthetic gene cluster for the azoxy antibiotic valanimycin. Mol. Microbiol. 46, 505-517. doi: 10.1046/j.1365-2958.2002.03169.x

Garg, R. P., and Parry, R. J. (2010). Regulation of valanimycin biosynthesis in Streptomyces viridifaciens: characterization of VlmI as a Streptomyces antibiotic regulatory protein (SARP). Microbiology 156(Pt 2), 472-483. doi: 10.1099/mic.0.033167-0

Gebetsberger, J., and Polacek, N. (2013). Slicing tRNAs to boost functional ncRNA diversity. RNA Biol. 10, 1798-1806. doi: 10.4161/rna.27177

Gebetsberger, J., Zywicki, M., Künzi, A., and Polacek, N. (2012). tRNA-derived fragments target the ribosome and function as regulatory non-coding RNA in Haloferax volcanii. Archaea 2012:260909. doi: 10.1155/2012/260909

Giannouli, S., Kyritsis, A., Malissovas, N., Becker, H. D., and Stathopoulos, C. (2009). On the role of an unusual tRNAGly isoacceptor in Staphylococcus aureus. Biochimie 91, 344-351. doi: 10.1016/j.biochi.2008.10.009

Givens, R. M., Lin, M. H., Taylor, D. J., Mechold, U., Berry, J. O., and Hernandez, V. J. (2004). Inducible expression, enzymatic activity, and origin of higher plant homologues of bacterial RelA/SpoT stress proteins in Nicotiana tabacum. J. Biol. Chem. 279, 7495-7504. doi: 10.1074/jbc.M311573200

Gonda, D. K., Bachmair, A., Wünning, I., Tobias, J. W., Lane, W. S., and Varshavsky, A. (1989). Universality and structure of the N-end rule. J. Biol. Chem. 264, 16700-16712.

Gondry, M., Sauguet, L., Belin, P., Thai, R., Amouroux, R., Tellier, C., et al. (2009). Cyclodipeptide synthases are a family of tRNA-dependent peptide bond-forming enzymes. Nat. Chem. Biol. 5, 414-420. doi: 10.1038/nchembio.175

Gorla, M., and Sepuri, N. B. (2014). Perturbation of apoptosis upon binding of tRNA to the heme domain of cytochrome c. Apoptosis 19, 259-268. doi: 10.1007/s10495013-0915-6

Graciet, E., Hu, R. G., Piatkov, K., Rhee, J. H., Schwarz, E. M., and Varshavsky, A. (2006). Aminoacyl-transferases and the N-end rule pathway of prokaryotic/eukaryotic specificity in a human pathogen. Proc. Natl. Acad. Sci. U.S.A. 103, 3078-3083. doi: 10.1073/pnas.0511224103

Green, N. J., Grundy, F. J., and Henkin T. M. (2010). The T box mechanism: tRNA as a regulatory molecule. FEBS Lett. 584, 318-324. doi: 10.1016/j.febslet.2009.11.056

Grundy, F. J., Yousef, M. R., and Henkin, T. M. (2005). Monitoring uncharged tRNA during transcription of the Bacillus subtilis glyQS gene. J. Mol. Biol. 346, 73-81. doi: 10.1016/j.jmb.2004.11.051

Hanada, T., Weitzer, S., Mair, B., Bernreuther, C., Wainger, B. J., Ichida, J., et al. (2013). CLP1 links tRNA metabolism to progressive motor-neuron loss. Nature 495, 474-480. doi: 10.1038/nature 1923

Harding, H. P., Novoa, I., Zhang, Y., Zeng, H., Wek R., Schapira, M., et al. (2000). Regulated translation initiation controls stress-induced gene expression in mammalian cells. Mol. Cell 6, 1099-1108. doi: 10.1016/S1097-2765(00) 00108-8

Haseltine, W. A., and Block, R. (1973). Synthesis of guanosine tetra- and pentaphosphate requires the presence of a codon-specific, uncharged transfer ribonucleic acid in the acceptor site of ribosomes. Proc. Natl. Acad. Sci. U.S.A. 70, 1564-1568. doi: 10.1073/pnas.70.5.1564

Haussecker, D., Huang, Y., Lau, A., Parameswaran, P., Fire, A. Z., Kay, M. A. et al. (2010). Human tRNA-derived small RNAs in the global regulation of RNA silencing. RNA 16, 673-695. doi: 10.1261/rna.2000810

Henkin, T. M., and Yanofsky, C. (2002). Regulation by transcription attenuation in bacteria: how RNA provides instructions for transcription termination/antitermination decisions. Bioessays 24, 700-707. doi: 10.1002/ bies. 10125

Hinnebusch, A. G. (2005). Translational regulation of GCN4 and the general amino acid control of yeast. Annu. Rev. Microbiol. 59, 407-450. doi: 10.1146/annurev.micro.59.031805.133833

Holcik, M., and Sonenberg, N. (2005). Translational control in stress and apoptosis. Nat. Rev. Mol. Cell. Biol. 6, 318-327. doi: 10.1038/nrm1618
Hou, Y. M., and Yang, X. (2013). Regulation of cell death by transfer RNA. Antioxid. Redox. Signal. 19, 583-594. doi: 10.1089/ars.2012.5171

Hsieh, L. C., Lin, S. I., Shih, A. C., Chen, J. W., Lin, W. Y., Tseng, C. Y., et al. (2009). Uncovering small RNA-mediated responses to phosphate deficiency in Arabidopsis by deep sequencing. Plant Physiol. 151, 2120-2132. doi: 10.1104/pp.109.147280

Ivanov, P., Emara, M. M., Villen, J., Gygi, S. P., and Anderson, P. (2011). Angiogenininduced tRNA fragments inhibit translation initiation. Mol. Cell 43, 613-623. doi: 10.1016/j.molcel.2011.06.022

Jia, J. M., Ma, X. C., Wu, C. F., Wu, L. J., and Hu, G. S. (2005). Cordycedipeptide A, a new cyclodipeptide from the culture liquid of Cordyceps sinensis (Berk.) Sacc. Chem. Pharm. Bull. (Tokyo) 53, 582-583. doi: 10.1248/cpb. 53.582

Kanoh, K., Kohno, S., Katada, J., Hayashi, Y., Muramatsu, M., and Uno, I. (1999). Antitumor activity of phenylahistin in vitro and in vivo. Biosci. Biotechnol. Biochem. 63, 1130-1133. doi: 10.1271/bbb.63.1130

Kanzaki, H., Yanagisawa, S., and Nitoda, T. (2004). Enzymatic synthesis of dehydro cyclo(His-Phe)s, analogs of the potent cell cycle inhibitor, dehydrophenylahistin, and their inhibitory activities toward cell division. Biosci. Biotechnol. Biochem. 68, 2341-2345. doi: 10.1271/bbb.68.2341

Kaufmann, G. (2000). Anticodon nucleases. Trends Biochem. Sci. 25, 70-74. doi: 10.1016/S0968-0004(99)01525-X

Kawaji, H., Nakamura, M., Takahashi, Y., Sandelin, A., Katayama, S., Fukuda, S., et al. (2008). Hidden layers of human small RNAs. BMC Genomics 9:157. doi: 10.1186/1471-2164-9-157

Klein, S., Lorenzo, C., Hoffmann, S., Walther, J. M., Storbeck, S., Piekarski, T., et al. (2009). Adaptation of Pseudomonas aeruginosa to various conditions includes tRNA-dependent formation of alanyl-phosphatidylglycerol. Mol. Microbiol. 71, 551-565. doi: 10.1111/j.1365-2958.2008.06562.x

Kohn, H., and Widger, W. (2005). The molecular basis for the mode of action of bicyclomycin. Curr. Drug Targets. Infect. Disord 5, 273-295. doi: $10.2174 / 1568005054880136$

Krammer, P. H., Arnold, R., and Lavrik, I. N. (2007). Life and death in peripheral T cells. Nat. Rev. Immunol. 7, 532-542. doi: 10.1038/nri2115

Lautru, S., Gondry, M., Genet, R., and Pernodet, J. L. (2002). The albonoursin gene cluster of $\mathrm{S}$ noursei biosynthesis of diketopiperazine metabolites independent of nonribosomal peptide synthetases. Chem. Biol. 9, 1355-1364. doi: 10.1016/S1074-5521(02)00285-5

Lee, Y. S., Shibata, Y., Malhotra, A., and Dutta, A. (2009). A novel class of small RNAs: tRNA-derived RNA fragments (tRFs). Genes Dev. 23, 2639-2649. doi: $10.1101 /$ gad. 1837609

Li, J., and Yuan, J. (2008). Caspases in apoptosis and beyond. Oncogene 27, 61946206. doi: 10.1038/onc.2008.297

Li, Z., Ender, C., Meister, G., Moore, P. S., Chang, Y., and John, B. (2012). Extensive terminal and asymmetric processing of small RNAs from rRNAs, snoRNAs, snRNAs, and tRNAs. Nucleic Acids Res. 40, 6787-6799. doi: 10.1093/nar/ gks307

Liu, X., Kim, C. N., Yang, J., Jemmerson, R., and Wang, X. (1996). Induction of apoptotic program in cell-free extracts: requirement for dATP and cytochrome c. Cell 86, 147-157. doi: 10.1016/S0092-8674(00) 80085-9

Lloyd, A. J., Gilbey, A. M., Blewett, A. M., De Pascale, G., El Zoeiby, A., Levesque, R. C., et al. (2008). Characterization of tRNA-dependent peptide bond formation by MurM in the synthesis of Streptococcus pneumoniae peptidoglycan. J. Biol. Chem. 283, 6402-6417. doi: 10.1074/jbc.M708105200

Lu, P. D., Harding, H. P., and Ron, D. (2004). Translation reinitiation at alternative open reading frames regulates gene expression in an integrated stress response. $J$. Cell. Biol. 167, 27-33. doi: 10.1083/jcb.200408003

Magnusson, L. U., Farewell, A., and Nyström T. (2005). ppGpp: a global regulator in Escherichia coli. Trends Microbiol. 13, 236-242. doi: 10.1016/j.tim. 2005.03.008

Magyar, A., Zhang, X., Kohn H., and Widger, W. R. (1996). The antibiotic bicyclomycin affects the secondary RNA binding site of Escherichia coli transcription termination factor Rho. J. Biol. Chem. 271, 25369-25374. doi: $10.1074 /$ jbc. 271.41 .25369

Marquet, R., Isel, C., Ehresmann, C., and Ehresmann, B. (1995). tRNAs as primer of reverse transcriptases. Biochimie 77, 113-124. doi: 10.1016/03009084(96)88114-4 
Marshall, L., and White, R. J. (2008). Non-coding RNA production by RNA polymerase III is implicated in cancer. Nat. Rev. Cancer 8, 911-914. doi: $10.1038 / \mathrm{nrc} 2539$

Marton, M. J., Crouch, D., and Hinnebusch, A. G. (1993). GCN1, a translational activator of GCN4 in Saccharomyces cerevisiae, is required for phosphorylation of eukaryotic translation initiation factor 2 by protein kinase GCN2. Mol. Cell. Biol. 13, 3541-3556.

Marton, M. J., Vazquez de Aldana, C. R., Qiu, H., Chakraburtty, K., and Hinnebusch, A. G. (1997). Evidence that GCN1 and GCN20, translational regulators of GCN4, function on elongating ribosomes in activation of eIF2alpha kinase GCN2. Mol. Cell. Biol. 17, 4474-4489.

Masaki, H., and Ogawa, T. (2002). The modes of action of colicins E5 and D, and related cytotoxic tRNases. Biochimie 84, 433-438. doi: 10.1016/S03009084(02)01425-6

Maute, R. L., Schneider, C., Sumazin, P., Holmes, A., Califano, A., Basso, K., et al. (2013). tRNA-derived microRNA modulates proliferation and the DNA damage response and is down-regulated in B cell lymphoma. Proc. Natl. Acad. Sci. U.S.A 110, 1404-1409. doi: 10.1073/pnas.1206761110

Mei, Y., Yong, J., Liu, H., Shi, Y., Meinkoth, J., Dreyfuss, G., et al. (2010). tRNA binds to cytochrome $\mathrm{c}$ and inhibits caspase activation. Mol. Cell 37, 668-678. doi: 10.1016/j.molcel.2010.01.023

Minelli, A., Grottelli, S., Mierla, A., Pinnen, F., Cacciatore, I., and Bellezza, I. (2012). Cyclo(His-Pro) exerts anti-inflammatory effects by modulating NFkappaB and Nrf2 signalling. Int. J. Biochem. Cell Biol. 44, 525-535. doi: 10.1016/j.biocel.2011.12.006

Mogk, A., Schmidt, R., and Bukau, B. (2007). The N-end rule pathway for regulated proteolysis: prokaryotic and eukaryotic strategies. Trends Cell Biol. 17, 165-172. doi: $10.1016 /$ j.tcb.2007.02.001

Musetti, R., Polizzotto, R., Vecchione, A., Borselli, S., Zulini, L., D’Ambrosio, M., et al. (2007). Antifungal activity of diketopiperazines extracted from Alternaria alternata against Plasmopara viticola: an ultrastructural study. Micron 38, 643650. doi: 10.1016/j.micron.2006.09.001

Nanamiya, H., Kasai, K., Nozawa, A., Yun, C. S., Narisawa, T., and Murakami, K. (2008). Identification and functional analysis of novel (p)ppGpp synthetase genes in Bacillus subtilis. Mol. Microbiol. 67, 291-304. doi: 10.1111/j.1365 2958.2007.06018.x

Nekrasov, M. P., Ivshina, M. P., Chernov, K. G., Kovrigina, E. A., Evdokimova, V M., and Thomas, A. A. (2003). The mRNA-binding protein YB-1 (p50) prevents association of the eukaryotic initiation factor eIF4G with mRNA and inhibits protein synthesis at the initiation stage. J. Biol. Chem. 278, 13936-13943. doi: 10.1074/jbc.M209145200

Nowacka, M., Strozycki, P. M., Jackowiak, P., Hojka-Osinska, A., Szymanski, M., and Figlerowicz, M. (2013). Identification of stable, high copy number medium-sized RNA degradation intermediates that accumulate in plants under non-stress conditions. Plant Mol. Biol. 83, 191-204. doi: 10.1007/s11103-0130079-3

Pavon-Eternod, M., Gomes, S., Geslain, R., Dai, Q., Rosner, M. R., and Pan, T. (2009) tRNA over-expression in breast cancer and functional consequences. Nucleic Acids Res. 37, 7268-7280. doi: 10.1093/nar/gkp787

Pavon-Eternod, M., Gomes, S., Rosner, M. R., and Pan, T. (2013). Overexpression of initiator methionine tRNA leads to global reprogramming of tRNA expression and increased proliferation in human epithelial cells. RNA 19, 461-466. doi: 10.1261/rna.037507.112

Peschel, A., Jack, R. W., Otto, M., Collins, L. V., Staubitz, P., Nicholson, G., et al. (2001). Staphylococcus aureus resistance to human defensins and evasion of neutrophil killing via the novel virulence factor $\mathrm{MprF}$ is based on modification of membrane lipids with 1-lysine. J. Exp. Med. 193, 1067-1076. doi: 10.1084/jem.193.9.1067

Phizicky, E. M., and Alfonzo, J. D. (2010). Do all modifications benefit all tRNAs? FEBS Lett. 584, 265-271. doi: 10.1016/j.febslet.2009.11.049

Potuschak, T., Stary, S., Schlögelhofer, P., Becker, F., Nejinskaia, V., and Bachmair, A. (1998). PRT1 of Arabidopsis thaliana encodes a component of the plant N-end rule pathway. Proc. Natl. Acad. Sci. U.S.A. 95, 7904-7908. doi: 10.1073/pnas.95.14.7904

Prasad, C. (1995). Bioactive cyclic dipeptides. Peptides 16, 151-164. doi 10.1016/0196-9781(94)00017-Z

Qiu, H., Dong, J., Hu, C., Francklyn, C. S., and Hinnebusch, A. G. (2001). The tRNA-binding moiety in GCN2 contains a dimerization domain that interacts with the kinase domain and is required for tRNA binding and kinase activation. EMBO J. 20, 1425-1438. doi: 10.1093/emboj/20.6.1425

Rai, R., and Kashina, A. (2005). Identification of mammalian arginyltransferases that modify a specific subset of protein substrates. Proc. Natl. Acad. Sci. U.S.A. 102, 10123-10128. doi: 10.1073/pnas.0504500102

Riedl, S. J., and Shi, Y. (2004). Molecular mechanisms of caspase regulation during apoptosis. Nat. Rev. Mol. Cell Biol. 5, 897-907. doi: 10.1038/nrm1496

Rodriguez, P. L., and Carrasco, L. (1992). Gliotoxin: inhibitor of poliovirus RNA synthesis that blocks the viral RNA polymerase 3Dpol. J. Virol. 66, 1971-1976.

Ross, W., Vrentas, C. E., Sanchez-Vazquez, P., Gaal, T., and Gourse, R. L. (2013). The magic spot: a ppGpp binding site on E. coli RNA polymerase responsible for regulation of transcription initiation. Mol. Cell 50, 420-429. doi: 10.1016/j.molcel.2013.03.021

Roy, H., Becker, H. D., Mazauric, M. H., and Kern, D. (2007). Structural elements defining elongation factor Tu mediated suppression of codon ambiguity. Nucleic Acids Res. 35, 3420-3430. doi: 10.1093/nar/gkm211

Roy, H., Dare, K., and Ibba, M. (2009). Adaptation of the bacterial membrane to changing environments using aminoacylated phospholipids. Mol. Microbiol. 71, 547-550. doi: 10.1111/j.1365-2958.2008.06563.x

Roy, H., and Ibba, M. (2008). RNA-dependent lipid remodeling by bacterial multiple peptide resistance factors. Proc. Natl. Acad. Sci. U.S.A. 105, 4667-4672. doi: $10.1073 /$ pnas.0800006105

Roy, H., and Ibba, M. (2009). Broad range amino acid specificity of RNA-dependent lipid remodeling by multiple peptide resistance factors. J. Biol. Chem. 284, 2967729683. doi: 10.1074/jbc.M109.046367

Royet, J., and Dziarski, R. (2007). Peptidoglycan recognition proteins: pleiotropic sensors and effectors of antimicrobial defences. Nat. Rev. Microbiol. 5, 264-277. doi: $10.1038 /$ nrmicro1620

Ruggero, K., Guffanti, A., Corradin, A., Sharma, V. K., De Bellis, G., Corti G., et al. (2014). Small noncoding RNAs in cells transformed by human T-cell leukemia virus type 1: a role for a tRNA fragment as a primer for reverse transcriptase. $J$. Virol. 88, 3612-3622. doi: 10.1128/JVI.02823-13

Saad, N. Y., Stamatopoulou, V., Brayé, M., Drainas, D., Stathopoulos, C., and Becker, H. D. (2013). Two-codon T-box riboswitch binding two tRNAs. Proc. Natl. Acad. Sci. U.S.A. 110, 12756-12761. doi: 10.1073/pnas.1304307110

Saikia, M., Krokowski, D., Guan, B. J., Ivanov, P., Parisien, M., Hu, G. F., et al. (2012). Genome-wide identification and quantitative analysis of cleaved tRNA fragments induced by cellular stress. J. Biol. Chem. 287, 42708-42725. doi: 10.1074/jbc.M112.371799

Sanderson, L. E., and Uhlenbeck, O. C. (2007). The 51-63 base pair of tRNA confers specificity for binding by EF-Tu. RNA 13, 835-840. doi: 10.1261/rna.485307

Sattlegger, E., and Hinnebusch, A. G. (2000). Separate domains in GCN1 for binding protein kinase GCN2 and ribosomes are required for GCN2 activation in amino acid-starved cells. EMBO J. 19, 6622-6633. doi: 10.1093/emboj/19. 23.6622

Sauguet, L., Moutiez, M., Li, Y., Belin, P., Seguin, J., Le Du, M. H., et al. (2011). Cyclodipeptide synthases, a family of class-I aminoacyl-tRNA synthetase-like enzymes involved in non-ribosomal peptide synthesis. Nucleic Acids Res. 39, 4475-4489. doi: 10.1093/nar/gkr027

Schaefer, M., Pollex, T., Hanna, K., Tuorto, F., Meusburger, M., Helm, M. T., etal. (2010). RNA methylation by Dnmt2 protects transfer RNAs against stress-induced cleavage. Genes Dev. 24, 1590-1595. doi: 10.1101/ gad. 586710

Schneider, T., Senn, M. M., Berger-Bächi, B., Tossi, A., Sahl, H. G., and Wiedemann, I. (2004). In vitro assembly of a complete, pentaglycine interpeptide bridge containing cell wall precursor (lipid II-Gly5) of Staphylococcus aureus. Mol. Microbiol. 53, 675-685. doi: 10.1111/j.1365-2958.2004.04149.x

Shepherd, J. (2011). Characterisation of Pneumococcal Peptidoglycan Cross-Linking Enzymology. Ph.D. thesis, Doctoral dissertation, University of Warwick.

Shepherd, J., and Ibba, M. (2013a). Direction of aminoacylated transfer RNAs into antibiotic synthesis and peptidoglycan-mediated antibiotic resistance. FEBS Lett. 587, 2895-2904. doi: 10.1016/j.febslet.2013.07.036

Shepherd, J., and Ibba, M. (2013b). Lipid II-independent trans editing of mischarged tRNAs by the penicillin resistance factor MurM. J. Biol. Chem. 288, 25915-25923. doi: 10.1074/jbc.M113.479824

Shrader, T. E., Tobias, J. W., and Varshavsky, A. (1993). The N-end rule in Escherichia coli: cloning and analysis of the leucyl, phenylalanyl-tRNA-protein transferase gene aat. J. Bacteriol. 175, 4364-4374. 
Sobala, A., and Hutvagner, G. (2011). Transfer RNA-derived fragments: origins, processing, and functions. Wiley Interdiscip. Rev. RNA 2, 853-862. doi: 10.1002/wrna.96

Sobala, A., and Hutvagner, G. (2013). Small RNAs derived from the $5^{\prime}$ end of tRNA can inhibit protein translation in human cells. RNA Biol. 10, 553-563. doi: 10.4161/rna.24285

Spriggs, K. A., Bushell, M., and Willis, A. E. (2010). Translational regulation of gene expression during conditions of cell stress. Mol. Cell 40, 228-237. doi: 10.1016/j.molcel.2010.09.028

Sreedhar, A. S., and Csermely, P. (2004). Heat shock proteins in the regulation of apoptosis: new strategies in tumor therapy: a comprehensive review. Pharmacol. Ther. 101, 227-257. doi: 10.1016/j.pharmthera.2003.11.004

Staubitz, P., Neumann, H., Schneider, T., Wiedemann, I., and Peschel, A. (2004). MprF-mediated biosynthesis of lysylphosphatidylglycerol, an important determinant in staphylococcal defensin resistance. FEMS Microbiol. Lett. 231, 67-71. doi: 10.1016/S0378-1097(03)00921-2

Strom, K., Sjögren, J., Broberg, A., and Schnürer, J. (2002). Lactobacillus plantarum MiLAB 393 produces the antifungal cyclic dipeptides cyclo(L-Phe-L-Pro) and cyclo(L-Phe-trans-4-OH-L-Pro) and 3-phenyllactic acid. Appl. Environ. Microbiol. 68, 4322-4327. doi: 10.1128/AEM.68.9.4322-4327.2002

Suto, K., Shimizu, Y., Watanabe, K., Ueda, T., Fukai, S., Nureki, O., et al. (2006). Crystal structures of leucyl/phenylalanyl-tRNA-protein transferase and its complex with an aminoacyl-tRNA analog. EMBO J. 25, 5942-5950. doi: 10.1038/sj.emboj.7601433

Sy, J., and Lipmann, F. (1973). Identification of the synthesis of guanosine tetraphosphate (MS I) as insertion of a pyrophosphoryl group into the $3^{\prime}$-position in guanosine 5'-diphosphate. Proc. Natl. Acad. Sci. U.S.A. 70, 306-309. doi: 10.1073/pnas.70.2.306

Tasaki, T., Sriram, S. M., Park, K. S., and Kwon, Y. T. (2012). The N-end rule pathway. Annu. Rev. Biochem. 81, 261-289. doi: 10.1146/annurev-biochem051710-093308

Thedieck, K., Hain, T., Mohamed, W., Tindall, B. J., Nimtz, M., Chakraborty, T. et al. (2006). The MprF protein is required for lysinylation of phospholipids in listerial membranes and confers resistance to cationic antimicrobial peptides (CAMPs) on Listeria monocytogenes. Mol. Microbiol. 62, 1325-1339. doi: 10.1111/j.13652958.2006.05452.x

Thompson, C. B. (1995). Apoptosis in the pathogenesis and treatment of disease. Science 267, 1456-1462. doi: 10.1126/science.7878464

Thompson, D. M., Lu, C., Green, P. J., and Parker, R. (2008). tRNA cleavage is a conserved response to oxidative stress in eukaryotes. RNA 14, 2095-2103. doi: 10.1261/rna.1232808

Thompson, D. M., and Parker, R. (2009). The RNase Rnylp cleaves tRNAs and promotes cell death during oxidative stress in Saccharomyces cerevisiae. J. Cell Biol. 185, 43-50. doi: 10.1083/jcb.200811119

Thornberry, N. A., and Lazebnik, Y. (1998). Caspases: enemies within. Science 281, 1312-1316. doi: 10.1126/science.281.5381.1312

Tobias, J. W., Shrader, T. E., Rocap, G., and Varshavsky, A. (1991). The N-end rule in bacteria. Science 254, 1374-1377. doi: 10.1126/science.1962196

Vattem, K. M., and Wek, R. C. (2004). Reinitiation involving upstream ORFs regulates ATF4 mRNA translation in mammalian cells. Proc. Natl. Acad. Sci. U.S.A. 101, 11269-11274. doi: 10.1073/pnas.0400541101

Vollmer, W., Blanot, D., and de Pedro, M. A. (2008). Peptidoglycan structure and architecture. FEMS Microbiol. Rev. 32, 149-167. doi: 10.1111/j.15746976.2007.00094.x

Wang, Q., Lee, I., Ren, J., Ajay, S. S., Lee, Y. S., and Bao, X. (2013). Identification and functional characterization of tRNA-derived RNA fragments (tRFs) in respiratory syncytial virus infection. Mol. Ther. 21, 368-379. doi: 10.1038/mt.2012.237

Wang, X. (2001). The expanding role of mitochondria in apoptosis. Genes Dev. 15, 2922-2933.
Waring, P., and Beaver, J. (1996). Cyclosporin A rescues thymocytes from apoptosis induced by very low concentrations of thapsigargin: effects on mitochondrial function. Exp. Cell Res. 227, 264-276. doi: 10.1006/excr. 1996.0276

Wek, S. A., Zhu, S., and Wek, R. C. (1995). The histidyl-tRNA synthetase-related sequence in the eIF-2 alpha protein kinase GCN2 interacts with tRNA and is required for activation in response to starvation for different amino acids. Mol. Cell Biol. 15, 4497-4506.

Winter, A. G., Sourvinos, G., Allison, S. J., Tosh, K., Scott, P. H., Spandidos, D. A., et al. (2000). RNA polymerase III transcription factor TFIIIC2 is overexpressed in ovarian tumors. Proc. Natl. Acad. Sci. U.S.A. 97, 12619-12624. doi: 10.1073/pnas.230224097

Yamasaki, S., Ivanov, P., Hu, G. F., and Anderson P. (2009). Angiogenin cleaves tRNA and promotes stress-induced translational repression. J. Cell Biol. 185, 35-42. doi: $10.1083 /$ jcb. 200811106

Yamato, M., Iinuma, H., Naganawa, H., Yamagishi, Y., Hamada, M., Masuda, T., etal. (1986). Isolation and properties of valanimycin, a new azoxy antibiotic. J. Antibiot. (Tokyo) 39, 184-191. doi: 10.7164/antibiotics. 39.184

Yeung, M. L., Bennasser, Y., Watashi, K., Le, S.-Y., Houzet, L., and Jeang K-T. (2009). Pyrosequencing of small non-coding RNAs in HIV-1 infected cells: evidence for the processing of a viral-cellular double-stranded RNA hybrid. Nucleic Acids Res. 37, 6575-6586. doi: 10.1093/nar/gkp707

Zhang, W., Ntai, I., Kelleher, N. L., and Walsh, C. T. (2011). tRNA-dependent peptide bond formation by the transferase PacB in biosynthesis of the pacidamycin group of pentapeptidyl nucleoside antibiotics. Proc. Natl. Acad. Sci. U.S.A. 108, 1224912253. doi: 10.1073/pnas. 1109539108

Zhang, W., Ostash, B., and Walsh, C. T. (2010). Identification of the biosynthetic gene cluster for the pacidamycin group of peptidyl nucleoside antibiotics. Proc. Natl. Acad. Sci. U.S.A. 107, 16828-16833. doi: 10.1073/pnas. 1011557107

Zhou, D., Palam, L. R., Jiang, L., Narasimhan, J., Staschke, K. A., and Wek, R. C. (2008). Phosphorylation of eIF2 directs ATF5 translational control in response to diverse stress conditions. J. Biol. Chem. 283, 7064-7073. doi: 10.1074/jbc.M708530200

Zhou, Y., Goodenbour, J. M., Godley, L. A., Wickrema, A., and Pan, T. (2009). High levels of tRNA abundance and alteration of tRNA charging by bortezomib in multiple myeloma. Biochem. Biophys. Res. Commun. 385, 160-164. doi: 10.1016/j.bbrc.2009.05.031

Zou, H., Henzel, W. J., Liu, X., Lutschg, A., and Wang X. (1997). Apaf-1, a human protein homologous to C. elegans CED-4, participates in cytochrome cdependent activation of caspase-3. Cell 90, 405-413. doi: 10.1016/S0092-8674(00) 80501-2

Conflict of Interest Statement: The authors declare that the research was conducted in the absence of any commercial or financial relationships that could be construed as a potential conflict of interest.

Received: 31 March 2014; accepted: 21 May 2014; published online: 11 June 2014. Citation: Raina $M$ and Ibba $M$ (2014) tRNAs as regulators of biological processes. Front. Genet. 5:171. doi: 10.3389/fgene.2014.00171

This article was submitted to Non-Coding RNA, a section of the journal Frontiers in Genetics.

Copyright (C) 2014 Raina and Ibba. This is an open-access article distributed under the terms of the Creative Commons Attribution License (CC BY). The use, distribution or reproduction in other forums is permitted, provided the original author(s) or licensor are credited and that the original publication in this journal is cited, in accordance with accepted academic practice. No use, distribution or reproduction is permitted which does not comply with these terms. 